\title{
Homotopy Invariance of the Space of Metrics with Positive Scalar Curvature on Manifolds with Singularities
}

Boris BOTVINNIK ${ }^{\mathrm{a}}$ and Mark G. WALSH ${ }^{\mathrm{b}}$

a) Department of Mathematics, University of Oregon, Eugene, OR, 97405, USA

E-mail: botvinn@uoregon.edu

URL: http://darkwing.uoregon.edu/ botvinn/

b) Department of Mathematics and Statistics, Maynooth University, Maynooth, Ireland

E-mail: Mark.Walsh@mu.ie

Received June 16, 2020, in final form March 24, 2021; Published online April 02, 2021

https://doi.org/10.3842/SIGMA.2021.034

\begin{abstract}
In this paper we study manifolds, $X_{\Sigma}$, with fibred singularities, more specifically, a relevant space $\mathcal{R}^{\mathrm{psc}}\left(X_{\Sigma}\right)$ of Riemannian metrics with positive scalar curvature. Our main goal is to prove that the space $\mathcal{R}^{\text {psc }}\left(X_{\Sigma}\right)$ is homotopy invariant under certain surgeries on $X_{\Sigma}$.
\end{abstract}

Key words: positive scalar curvature metrics; manifolds with singularities; surgery

2020 Mathematics Subject Classification: 53C27; 57R65; 58J05; 58J50

\section{Introduction}

\subsection{Existence of a psc-metric}

A classical result in this subject concerns the existence of metrics of positive scalar curvature (psc-metrics) on a simply-connected smooth closed manifold $M$. There are two cases here: either $M$ is a spin manifold or it is not. Recall that in the case when $M$ is spin, there is an index $\alpha(M) \in K O^{-n}$ of the Dirac operator valued in real $K$-theory. Here is the result:

Theorem 1.1 (Gromov-Lawson [12], Stolz [15]). Let $M$ be a smooth closed simply connected manifold of dimension $n \geq 5$.

(i) If $M$ is spin, then $M$ admits a psc-metric if and only if the index $\alpha(M) \in K O^{-n}$ of the Dirac operator on $M$ vanishes.

(ii) If $M$ is not spin, then $M$ always admits a psc-metric.

We denote by $\mathcal{R}^{\mathrm{psc}}(M)$ the space of psc-metrics on $M$. Recall that one of the major tools used to prove Theorem 1.1 is the surgery technique due to Gromov and Lawson (proved independently by Schoen and Yau). In particular, Gromov-Lawson observed that a psc-metric survives surgeries of codimension at least three (such surgeries are called admissible). It turns out that the homotopy type of the space $\mathcal{R}^{\mathrm{psc}}(M)$ is invariant under such surgeries; see [8, 9, 18].

This paper is a contribution to the Special Issue on Scalar and Ricci Curvature in honor of Misha Gromov on his 75th Birthday. The full collection is available at https://www.emis.de/journals/SIGMA/Gromov.html 


\subsection{Existence of a psc-metric on a manifold with Baas-Sullivan singularities}

We start with the simplest case, where the geometrical picture is transparent. Let $\left(L, g_{L}\right)$ be a closed Riemannian manifold, in which the metric $g_{L}$ is assumed to have zero scalar curvature. Let $Y$ be a closed smooth manifold, such that the product $Y \times L$ is a boundary of a smooth manifold $X$, i.e., $\partial X=Y \times L$. Here is a natural geometrical question:

Question 1. Does there exist a psc-metric $g_{Y}$ on $Y$, such that the product metric $g_{Y}+g_{L}$ on $\partial X=Y \times L$ can be extended (being a product near $\partial X$ ) to a psc-metric $g_{X}$ on $X$ ?

It is convenient to denote $\beta X:=Y$ (which is sometimes called the Bockstein manifold of $X$ ). Thus, for now, the boundary of $X$ forms the total space of a trivial bundle,

$$
\partial X=\beta X \times L \rightarrow \beta X .
$$

In this case, a manifold with Baas-Sullivan singularities of the type L (or just L-singularities), and denoted $X_{\Sigma}$, is obtained as

$$
X_{\Sigma}:=X \cup_{\partial X}-\beta X \times C(L),
$$

where $C(L)$ is a cone over $L$ (and the minus sign represents a change of orientation in the case when manifolds are oriented).

Assume for a moment that there is a psc-metric $g_{X}$ on $X$ such that $g_{X}$ is a product metric of the form $g_{\partial X}+\mathrm{d} t^{2}$, near $\partial X$, where the boundary metric $g_{\partial X}:=\left.g_{X}\right|_{\partial X}$ satisfies $g_{\partial X}=$ $g_{\beta X}+g_{L}$. Then we would like to extend the metric $g_{X}$ to a psc-metric (with singularities) on $X_{\Sigma}$. (For brevity we will use the term metric here with the understanding that on $X_{\Sigma}$ (or on $C(L)$ ), metrics are necessarily singular.) To do this, we begin by extending the scalar-flat metric, $g_{L}$, to a scalar-flat metric on the cone $C(L)$, which we denote $g_{C(L)}$. There is an obvious way to do this and details are provided in the appendix.

To obtain the desired metric on $X_{\Sigma}$, it will be necessary to attach this conical metric, $g_{C(L)}$, to a cylindrical metric $\mathrm{d} t^{2}+g_{L}$. While these metrics do not attach smoothly, it is possible to bend one end of the cylinder inwards so that it matches the conical metric. This process creates some positive but no negative scalar curvature. With this in mind, we construct an "attaching" metric of non-negative scalar curvature on the cylinder $X \times[0,1]$ which takes the form $\mathrm{d} t^{2}+g_{L}$ at one end and smoothly attaches to $g_{C(L)}$ at the other. This metric is denoted $g_{\text {att }(L)}$ and details of its construction are given in the appendix.

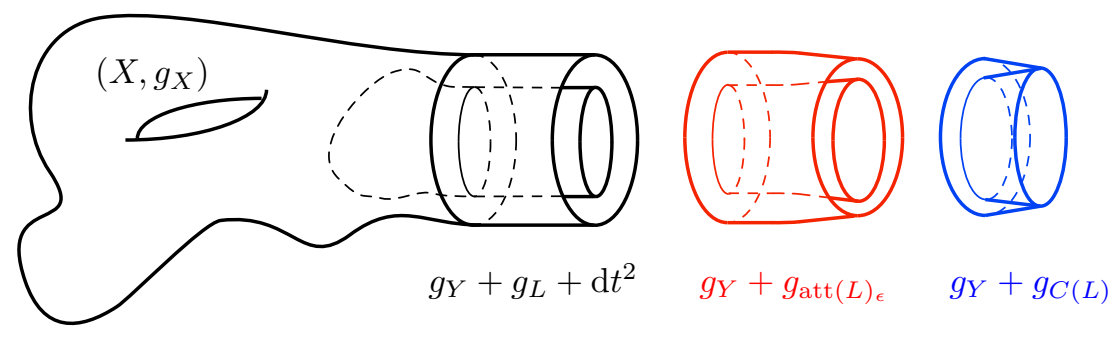

Figure 1. Gluing together the components, $g_{X}, g_{Y}+g_{\text {att }(L)}$ and $g_{Y}+g_{C(L)}$ of the metric $g_{X_{\Sigma}}$.

Remark 1.2. We emphasize that, according to our construction, the metrics $g_{\text {att }(L)}$ and $g_{C(L)}$ are both invariant under the action of the isometry group Iso $\left(g_{L}\right)$ of the metric $g_{L}$. In our work, the role of $L$ will be played by either the circle $S^{1}$ (with $U(1)$ as the isometry group), some other appropriate Lie group or a homogeneous space, such as $S^{n}$ or $\mathbb{H} \mathbb{P}^{n}$, equipped with the natural metric $g_{L}$ of non-negative constant scalar curvature. 
Returning now to the manifold $X_{\Sigma}$, we define a metric $g_{X_{\Sigma}}$ by

$$
g_{X_{\Sigma}}:=g_{X} \cup\left(g_{Y}+g_{\text {att }(L)}\right) \cup\left(g_{Y}+g_{C(L)}\right),
$$

obtained by gluing in the obvious way; see Figure 1 . The following fact is an easy consequence of Proposition 6.2.

Proposition 1.3. The construction of $g_{X_{\Sigma}}$ above yields a smooth (singular) metric of nonnegative scalar curvature on $X_{\Sigma}$.

The above construction motivates the notion of a well-adapted metric on $X_{\Sigma}$. Before stating it, we fix a scalar-flat metric $g_{L}$ on $L$ and equip $X$ with a boundary collar $c: \partial X \times[-1,1] \rightarrow X$, where $c(\partial X \times\{-1\})=\partial X$.

We say that a metric $g$ on $X_{\Sigma}$ is a well-adapted Riemannian metric on $X_{\Sigma}$ if

(i) the restriction $\left.g\right|_{X}$ satisfies the following conditions on the collar $c$ :

$$
\left.c^{*} g\right|_{\partial X \times[0,1]}=g_{\beta X}+g_{L}+\mathrm{d} t^{2} \quad \text { and }\left.\quad c^{*} g\right|_{\partial X \times[-1,0]}=g_{\beta X}+g_{\mathrm{att}(L)},
$$

(ii) the restriction $\left.g\right|_{\beta X \times C(L)}$ splits as a product-metric $\left.g\right|_{\beta X \times C(L)}=g_{\beta X}+g_{C(L)}$.

We denote by $\mathcal{R}\left(X_{\Sigma}\right)$ the space of all well-adapted Riemannian metrics on $X_{\Sigma}$, and by $\mathcal{R}^{\mathrm{psc}}\left(X_{\Sigma}\right)$ its subspace of psc-metrics. Thus, Question 1 above is equivalently asking whether the space $\mathcal{R}^{\text {psc }}\left(X_{\Sigma}\right)$ is non-empty. This existence question was addressed and even affirmatively resolved for some particular examples of the singularity types $L$ (provided that all manifolds involved are spin and both $X$ and $\beta X$ are simply-connected; see [3]).

There is a particularly interesting example here. Let us consider spin manifolds, and choose $L=S^{1}$ with a non-trivial spin structure, so that $L$ represents the generator $\eta \in \Omega_{1}^{\text {spin }}=\mathbb{Z}_{2}$. We denote by $\Omega_{*}^{\text {spin, }, \eta}(-)$ the bordism theory of spin manifolds with $\eta$-singularities, and by MSpin ${ }^{\eta}$ the corresponding representing spectrum. We refer to [3] for details on cobordism theory $\Omega_{*}^{\text {spin, }, \eta}(-)$.

It turns out, there exists a Dirac operator on spin-manifolds with $\eta$-singularities. Furthermore, there is a natural transformation $\alpha^{\eta}: \Omega_{*}^{\text {spin, } \eta} \rightarrow K O_{*}^{\eta}$ which evaluates the index of that Dirac operator, where the " $K$-theory with $\eta$-singularities" $K O_{*}^{\eta}(-)$ coincides with the usual complex $K$-theory. Here is the result from [3]:

Theorem 1.4 (Botvinnik [3]). Let $X$ be a simply connected spin manifold with nonempty $\eta$ singularity of dimension $n \geq 7$. Then $X$ admits a metric of positive scalar curvature if and only if $\alpha^{\eta}([X])=0$ in the group $K O_{n}^{\eta} \cong K U_{n}$.

\subsection{Existence of a psc-metric on a manifold with fibred singularities}

There are more general objects, namely, "manifolds with fibred singularities" (or pseudo-manifolds with singularities of depth one). Here again, we start with a manifold $X$ with boundary $\partial X \neq \varnothing$, which is the total space of a fibre bundle $\partial X \rightarrow \beta X$ with the fibre $L$. Usually the manifold $L$ is referred to as the link. To get geometrically interesting objects, we fix the following data on the manifold $L$ :

(a) a metric $g_{L}$ on $L$ of non-negative constant scalar curvature,

(b) a subgroup $G$ of the isometry group $\operatorname{Isom}\left(g_{L}\right)$ of the metric $g_{L}$. 
In particular, if $G$ is a nontrivial subroup, the metric $g_{L}$ is assumed to be homogeneous. Henceforth, we assume that the bundle $\partial X \rightarrow \beta X$ is a $G$-bundle; thus the bundle $\partial X \rightarrow \beta X$ is induced by a structure map $f: \beta X \rightarrow B G$.

Let $C(L)$ be a cone over $L$ with a cone metric, $g_{C(L)}$, which restricts to $g_{L}$ on the base and is scalar-flat. As we mentioned in Remark 1.2, the attaching metric $g_{\text {att }(L)}$ and the cone metric $g_{C(L)}$ are both invariant under the action of the isometry group $\operatorname{Isom}\left(g_{L}\right)$. In particular, the isometry action of $G$ on $L$ extends automatically to $C(L)$ and this gives rise to a fibre bundle $N(\beta X) \rightarrow \beta X$, obtained by "inserting" the cone $C(L)$ as a fibre with the same structure group $G$. Thus, we obtain a manifold with fibred singularities, $X_{\Sigma}$, given as $X_{\Sigma}:=X \cup_{\partial X}-N(\beta X)$. Such a manifold is referred to as an $(L, G)$-manifold.

Now we consider spin $(L, G)$-manifolds $X_{\Sigma}:=X \cup_{\partial X}-N(\beta X)$; this means that we first fix a spin structure on $L$, and $X$ and $\beta X$ are given spin structures which are respected by the bundle map $\partial X \rightarrow \beta X$. We will assume that the isometry group $G$ preserves the fixed spin structure on $L$. For short, we call such a bundle map $\partial X \rightarrow \beta X$ a geometric L-bundle.

There is a natural concept of spin cobordism between $(L, G)$-manifolds. Namely, we say that $W_{\Sigma}: X_{\Sigma} \rightsquigarrow X_{\Sigma, 1}$ is a $\operatorname{spin}(L, G,-\mathrm{fb})$-cobordism between

$$
X_{\Sigma}=X \cup_{\partial X}-N(\beta X) \quad \text { and } \quad X_{\Sigma, 1}=X_{1} \cup_{\partial X_{1}}-N\left(\beta X_{1}\right),
$$

if $W_{\Sigma}:=W \cup_{\partial^{s} W}-N(\beta W)$, where the boundary $\partial W$ is decomposed as

$$
\partial W=X \cup \partial^{s} W \cup X_{1},
$$

and $\partial^{s} W: \partial X \rightsquigarrow \partial X_{1}$ is a spin cobordism over a geometric $L$-bundle map $p: \partial^{s} W \rightarrow \beta W$ which restricts to given geometric $L$-bundles $\partial X \rightarrow \beta X$ and $\partial X_{1} \rightarrow \beta X_{1}$. This gives a corresponding cobordism theory $\Omega_{n}^{\mathrm{spin},(L, G)-\mathrm{fb}}(-)$; see $[5,6,7]$ for more details.

It turns out there are several interesting cases when the Dirac operator is well defined on such spin $(L, G)$-manifolds, and the index of such operator gives a homomorphism

$$
\alpha^{(L, G)-\mathrm{fb}}: \Omega_{n}^{\mathrm{spin},(L, G)-\mathrm{fb}} \rightarrow K O_{n}^{(L, G)-\mathrm{fb}},
$$

where $K O_{n}^{(L, G) \text {-fb }}$ is an appropriate $K$-theory; see [5, 6, 7] and the examples below in this section.

We have to modify the above definition of well-adapted Riemannian metric on $X_{\Sigma}$ (we give a detailed definition in Section 2). Roughly, in this setting, a well-adapted Riemannian metric $g$ on $X_{\Sigma}=X \cup_{\partial X}-N(\beta X)$ is a regular Riemannian metric restricted to $X$ (satisfying certain product conditions near the boundary), and the restriction metric $\left.g\right|_{N(\beta X)}$ is determined by a requirement that the projection $N(\beta X) \rightarrow \beta X$ is a Riemannian submersion (which has a structure group $\left.G \subset \operatorname{Isom}\left(g_{L}\right)\right)$ and with the cone metric $g_{C(L)}$ on the fibre. We denote by $\mathcal{R}\left(X_{\Sigma}\right)$ the space of well-adapted Riemannian metrics on $X_{\Sigma}$, and by $\mathcal{R}^{\text {psc }}\left(X_{\Sigma}\right)$ its subspace of psc-metrics; see Section 2.4 for a precise definition of this subspace. Below we describe some interesting cases.

1.3.1. We assume that all $(L, G)$-manifolds are spin, $L=S^{1}$ representing $\eta \in \Omega_{1}^{\text {spin }}$, and $G=S^{1}$. We obtain a corresponding bordism group $\Omega_{*}^{\text {spin, } \eta \text {-fb }}$ of such manifolds. Then there exists an appropriate Dirac operator on $X_{\Sigma}$, and index map $\alpha^{\eta-\mathrm{fb}}: \Omega_{*}^{\text {spin, } \eta \text {-fb }} \rightarrow K O_{*}^{\eta-\mathrm{fb}}$ evaluating the index of that Dirac operator. Here is the existence result for psc-metrics in that setting:

Theorem 1.5 (Botvinnik-Rosenberg [7]). Let $X_{\Sigma}=X \cup N(\beta X)$ be a simply connected spin manifold with fibred $\eta$-singularity (i.e., $X$ and $\beta X$ are simply-connected and spin) of dimension $n \geq 7$. Then $\mathcal{R}^{\mathrm{psc}}\left(X_{\Sigma}\right) \neq \varnothing$ if and only if $\alpha^{\eta-\mathrm{fb}}\left(\left[X_{\Sigma}\right]\right)=0$ in the group $K O_{n}^{\eta-\mathrm{fb}}$.

1.3.2. Again, we assume that all $(L, G)$-manifolds are spin: here $L$ is equipped with a metric $g_{L}$ with constant scalar curvature $s_{L}=\ell(\ell-1), \operatorname{dim} L=\ell$, and $G$ is a subgroup of the isometry group 
of the metric $g_{L}$. We assume $L=\partial \bar{L}$, where $\bar{L}$ is a smooth compact manifold with boundary, and the $G$-action on $L$ extends to a $G$-action on $\bar{L}$. In this setting, an $(L, G)$-manifold $X_{\Sigma}$ could be described as a triple $(X, \beta X, f)$, where $X$ is a manifold with boundary $\partial X$, which is a total space of a fibre bundle $\partial X \rightarrow \beta X$ (with a fibre $L$ and a structure group $G$ ) given by a map $f: \beta X \rightarrow B G$. In this setting we have indices $\alpha(\beta X) \in K O_{n-\ell-1}$ and $\alpha_{\text {cyl }}(X) \in K O_{n}$, where $n=\operatorname{dim} X$.

Here are the existence results:

Theorem 1.6 (Botvinnik-Piazza-Rosenberg [5]). Let $(X, \partial X, f)$ define a closed $(L, G)$-singular spin manifold $X_{\Sigma}$. Assume that $X, \beta X$, and $G$ are all simply connected, that $n-\ell \geq 6$, and suppose that $L$ is a spin boundary, say $L=\partial \bar{L}$, with the standard metric $g_{L}$ on $L$ extending to a psc-metric on $\bar{L}$, and with the $G$-action on $L$ extending to a $G$-action on $\bar{L}$. Assume that the two obstructions $\alpha(\beta X) \in K O_{n-\ell-1}$ and $\alpha_{\mathrm{cyl}}(X) \in K O_{n}$ both vanish. Then $X_{\Sigma}$ admits a well-adapted psc-metric.

Theorem 1.7 (Botvinnik-Piazza-Rosenberg [5]). Let $(X, \beta X, f)$ define a closed $(L, G)$-singular spin manifold $X_{\Sigma}$, with $L=\mathbb{H} \mathbb{P}^{2 k}$ and $G=\operatorname{Sp}(2 k+1), n \geq 1$. Assume that $\partial X=\beta X \times L$, i.e., the L-bundle over $\beta X$ is trivial, or in other words that the singularities are of Baas-Sullivan type. Then if $X$ and $\beta X$ are both simply connected and $n-8 k \geq 6, X_{\Sigma}$ has an adapted psc-metric if and only if the $\alpha$-invariants $\alpha(\beta X) \in K O_{n-8 k-1}$ and $\alpha_{\mathrm{cyl}}(X) \in K O_{n}$ both vanish.

There are several other interesting cases and also more general results when $X_{\Sigma}$ has nontrivial fundamental group; see [6]. Now we are ready to address our main result concerning homotopy invariance of the corresponding spaces of psc-metrics on $X_{\Sigma}$.

\subsection{Main result}

The homotopy-invariance of certain spaces of psc-metrics is a crucial property which has allowed detection of their non-trivial homotopy groups. Let $M$ be a closed spin manifold. An important consequence of the results due to Chernysh [8], Walsh [18, 19, 20] (see also recent work by Ebert and Frenck [9]) is that the homotopy type of the space $\mathcal{R}^{\mathrm{psc}}(M)$ is an invariant of the bordism class $[M] \in \Omega_{n}^{\text {spin }}$ (provided $M$ is simply-connected and $\left.n \geq 5\right) .{ }^{1}$

Notice that if $X_{\Sigma}=X \cup_{\partial X}-N(\beta X)$ is a pseudo-manifold with $(L, G)$-singularities equipped with structure map $f: \beta X \rightarrow B G$, then there are two types of surgery possible on $X$ :

(i) a surgery on its "resolution", i.e., the interior $X \subset X_{\Sigma}$ away from the boundary $\partial X$,

(ii) a surgery on the structure map $f: \beta X \rightarrow B G$.

In case $(i)$ all constructions are the same as in the case of closed manifolds; however, in case (ii), we have to be more careful. Indeed, let $\bar{B}: \beta X \rightsquigarrow \beta X_{1}$ be the trace of a surgery on the map $f: \beta X \rightarrow B G$, with $\partial \bar{B}=\beta X \sqcup-\beta X_{1}$ and $\beta X_{1}$, the manifold obtained from $\beta X$ by surgery on $f$. Then the map $f$ extends to a map $\bar{f}: \bar{B} \rightarrow B G$ which gives a fibre bundle $\bar{p}: Z \rightarrow \bar{B}$ with the fibre $L$. This gives us a new manifold $X_{1}=X \cup_{\partial X} Z$ with boundary $\partial X_{1}$, the total space over a new Bockstein $\beta X_{1}$ with the same fibre $L$. Also we obtain a new conical part $N\left(\beta X_{1}\right)$ as above. All of this results in a new pseudo-manifold

$$
X_{\Sigma, 1}=X_{1} \cup_{\partial X_{1}}-N\left(\beta X_{1}\right), \quad X_{1}=X \cup_{\partial X} Z
$$

with structure map $f_{1}=\left.\bar{f}\right|_{\beta X_{1}}: \beta X_{1} \rightarrow B G$. Here is our main technical result:

Theorem 1.8. Let $X_{\Sigma}=X \cup_{\partial X}-N(\beta X)$ be a pseudo-manifold with $(L, G)$-singularities, with $\operatorname{dim} X=n, \operatorname{dim} L=\ell$.

\footnotetext{
${ }^{1}$ There is also a similar result for non-simply connected manifolds.
} 
(i) Let $i: S^{p} \subset X$ be a sphere with trivial normal bundle, and $X_{\Sigma}^{\prime}$ be the result of surgery on $X_{\Sigma}$ along $S^{p}$. Then if $2 \leq p \leq n-3$, the spaces $\mathcal{R}^{\mathrm{psc}}\left(X_{\Sigma}\right)$ and $\mathcal{R}^{\mathrm{psc}}\left(X_{\Sigma}^{\prime}\right)$ are weakly homotopy equivalent.

(ii) Let $i: S^{p} \subset \beta X$ be a sphere with trivial normal bundle, and with $f \circ \iota: S^{p} \rightarrow B G$ homotopic to zero. Let $\bar{B}$ be a trace of the surgery along $S^{p} \subset \beta X$ with $\partial \bar{B}=\beta X \sqcup-\beta X_{1}$ and a structure map $\bar{f}: \bar{B} \rightarrow B G$. Then if $2 \leq p \leq n-\ell-3$, the spaces $\mathcal{R}^{\mathrm{psc}}\left(X_{\Sigma}\right)$ and $\mathcal{R}^{\mathrm{psc}}\left(X_{\Sigma, 1}\right)$ are homotopy equivalent, where $X_{\Sigma, 1}$ is given by (1.1).

Remark 1.9. As it deals with surgery on the interior, part $(i)$ of Theorem 1.8 above follows directly from Chernysh's theorem [8]. Our contribution in this paper is in proving part (ii).

Theorem 1.8 could be applied to a variety of interesting examples. Among these are:

(1) Let $L=\langle k\rangle$ be the set of $k$ points, and let $G=\mathbb{Z}_{k}$ be its "isometry group". Then a $\left(\langle k\rangle, \mathbb{Z}_{k}\right)$-manifold $X_{\Sigma}=X \cup_{\partial X}-N(\beta X)$ is assembled out of a manifold $X$ with boundary $\partial X$ equipped with a free $\mathbb{Z}_{k}$-action, and a structure map $\beta X \rightarrow B \mathbb{Z}_{k}$ classifies the corresponding $k$-folded covering $\partial X \rightarrow \beta X=\partial X / \mathbb{Z}_{k}$. Here $N(\beta X)$ is given by inserting the cone $C\langle k\rangle$ instead of $\langle k\rangle$ in the fibre bundle $\partial X \rightarrow \beta X$. Assuming that all manifolds are spin, we obtain corresponding bordism groups $\Omega_{*}^{\text {spin, },\left(\langle k\rangle, \mathbb{Z}_{k}\right)-\mathrm{fb}}$ and the corresponding

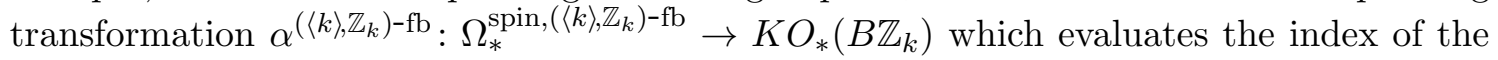
corresponding Dirac operator; see [7] for details.

(2) Let $\eta \in \Omega_{1}^{\text {spin }}$ be as above, i.e., $[L]=\eta$ and $G=S^{1}$. Then, similarly, we arrive at the bordism groups $\Omega_{*}^{\text {spin, } \eta \text {-fb }}$ and the index map $\alpha^{\eta-\mathrm{fb}}: \Omega_{*}^{\mathrm{spin}, \eta-\mathrm{fb}} \rightarrow K O_{*}^{\eta-\mathrm{fb}}$, as in Theorem 1.4 as above; see $[5,7]$ for details.

The above examples lead to the following two corollaries of Theorem 1.8:

Corollary 1.10. Let $X_{\Sigma}$ be a spin $(\langle k\rangle-\mathrm{fb})$-manifold. Assume $\operatorname{dim} X \geq 7$ and that $X$ and $\beta X$ are simply-connected. Then the homotopy type of the space $\mathcal{R}^{\mathrm{psc}}\left(X_{\Sigma}\right)$ is a bordism invariant and depends only on the bordism class $\left[X_{\Sigma}\right] \in \Omega_{n}^{\mathrm{spin},\langle k\rangle-\mathrm{fb}}$.

Corollary 1.11. Let $X_{\Sigma}$ be a spin ( $\eta$-fb)-manifold. Assume $\operatorname{dim} X \geq 9$ and that $X$ and $\beta X$ are simply-connected. Then the homotopy type of the space $\mathcal{R}^{\mathrm{psc}}\left(X_{\Sigma}\right)$ is a bordism invariant and depends only on the bordism class $\left[X_{\Sigma}\right] \in \Omega_{n}^{\mathrm{spin}, \eta-\mathrm{fb}}$.

Remark 1.12. To simplify the presentation, we consider only the spin case here. However, there are also non-spin cases where similar results hold. We leave this intentionally outside of this paper.

The cases addressed in Theorems 1.6 and 1.7 give interesting implications.

(3) Let $L$ and $G$ be as in Theorem 1.6, i.e., $G$ is a simply connected Lie group, $L$ is a spin boundary, say $L=\partial \bar{L}$, with the standard metric $g_{L}$ on $L$ extending to a psc-metric on $\bar{L}$, and with the $G$-action on $L$ extending to a $G$-action on $\bar{L}$. Then an $(L, G)$-singular spin manifold $X_{\Sigma}$ determines an element in the relevant bordism group $\Omega_{n}^{\text {spin, }(L, G)-\mathrm{fb}}$ which fits to an exact triangle (see [5]):

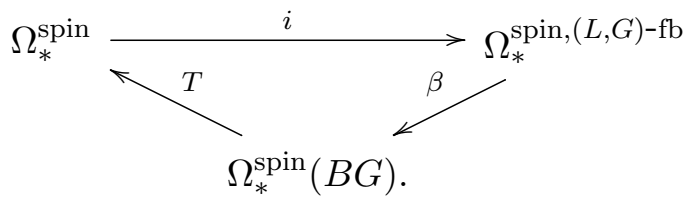

Here the indices $\alpha(\beta X) \in K O_{n-\ell-1}$ and $\alpha_{\text {cyl }}(X) \in K O_{n}$ can be thought of as homomor-

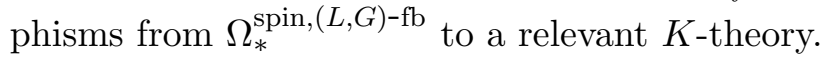


(4) Let $L=\mathbb{H}^{2 k}$ and $G=\operatorname{Sp}(2 k+1), n \geq 1$. Assume that $\partial X=\beta X \times L$, i.e., the $L$-bundle over $\beta X$ is trivial, or in other words that the singularities are of Baas-Sullivan type. Then a closed $(L, G)$-singular spin manifold $X_{\Sigma}$ determines an element in the corresponding bordism group $\Omega_{n}^{\text {spin, }(L, G) \text {-fb }}$, and, as above, the index homomorphism from $\Omega_{*}^{\text {spin },(L, G) \text {-fb }}$ to a relevant $K$-theory.

These examples lead to following corollary

Corollary 1.13. In both of the cases described in (3) and (4), the homotopy type of the space $\mathcal{R}^{\mathrm{psc}}\left(X_{\Sigma}\right)$ is a bordism invariant and depends on the bordism class $\left[X_{\Sigma}\right] \in \Omega_{n}^{\mathrm{spin},(L, G)-\mathrm{fb}}$, provided $n-\ell \geq 6$ (where $\ell=2 k$ in the case $(4)$ ).

Remark 1.14. To see how Theorem 1.8 implies the corollaries, it is enough to notice that in all those cases, the dimensional assumptions imply that a cobordism $W_{\Sigma}: X_{\Sigma} \rightsquigarrow X_{\Sigma, 1}$ could be modified so that the embedding $X_{\Sigma, 1} \subset W_{\Sigma}$ is two-connected in an appropriate sense, i.e., the induced embeddings $\beta X_{1} \subset \beta W$ and $\left(X_{1}, \partial X_{1}\right) \hookrightarrow(W, \partial W)$ are both 2-connected. Then the argument goes exactly as in the case of closed manifolds.

In the last section we show that the cases (3) and (4) above lead to an interesting result concerning homotopy groups of $\mathcal{R}^{\mathrm{psc}}\left(X_{\Sigma}\right)$.

\section{Preliminaries}

\subsection{Positive scalar curvature on manifolds with boundary}

Here we recall the main constructions and results from [20]. The set-up is as follows. Given a smooth compact $n$-dimensional manifold $X$ (possibly with boundary $\partial X \neq \varnothing$ ), we denote by $\mathcal{R}(X)$, the space of all Riemannian metrics on $X$. The space $\mathcal{R}(X)$ is equipped with the standard $C^{\infty}$-topology, giving it the structure of a Fréchet manifold; see [16, Chapter 1] for details. For each metric $g \in \mathcal{R}(X)$, we denote by $s_{g}: X \rightarrow \mathbb{R}$ the scalar curvature on $X$ of the metric $g$ and by $\mathcal{R}^{\mathrm{psc}}(X) \subset \mathcal{R}(X)$ the subspace of psc-metrics on $X$.

In the case when $\partial X \neq \varnothing$, it is necessary to consider certain subspaces of $\mathcal{R}^{\mathrm{psc}}(X)$, where metrics satisfy particular boundary constraints. With this in mind, we specify a collar embedding $c: \partial X \times[0,2) \hookrightarrow X$ around $\partial X$ and define the space $\mathcal{R}^{\mathrm{psc}}(X, \partial X)$ as:

$$
\mathcal{R}^{\mathrm{psc}}(X, \partial X):=\left\{h \in \mathcal{R}^{\mathrm{psc}}(X):\left.c^{*} h\right|_{\partial X \times I}=\left.h\right|_{\partial X}+\mathrm{d} t^{2}\right\},
$$

where $I:=[0,1] \subset[0,2)$. Fixing a particular metric $g \in \mathcal{R}^{\mathrm{psc}}(\partial X)$, we define the subspace $\mathcal{R}^{\mathrm{psc}}(X, \partial X)_{g} \subset \mathcal{R}^{\mathrm{psc}}(X, \partial X)$ of all psc-metrics $h \in \mathcal{R}^{\mathrm{psc}}(X, \partial X)$, where $\left.\left(c^{*} h\right)\right|_{\partial X \times\{0\}}=g$.

Let $Z: Y_{0} \rightsquigarrow Y_{1}$ be a bordism between $(n-1)$-dimensional manifolds $Y_{0}$ and $Y_{1}$ given together with collars $c_{i}: Y_{i} \times[0,2) \hookrightarrow Z, i=0,1$ near the boundary $\partial Z=Y_{0} \sqcup Y_{1}$. Then $\mathcal{R}^{\mathrm{psc}}(Z, \partial Z)$ denotes the space of psc-metrics on $Z$ which restrict as a product structure on the neighbourhood $c_{i}\left(Y_{i} \times I\right) \subset Z, i=0,1$; i.e., $c_{i}^{*} \bar{g}=g_{i}+\mathrm{d} t^{2}$ on $Y_{i} \times I$ for some pair of metrics $g_{i} \in \mathcal{R}^{\mathrm{psc}}\left(Y_{i}\right)$, $i=0,1$. Now we fix a pair of psc-metrics $g_{0} \in \mathcal{R}^{\mathrm{psc}}\left(Y_{0}\right)$ and $g_{1} \in \mathcal{R}^{\mathrm{psc}}\left(Y_{1}\right)$ and consider the following subspace of $\mathcal{R}^{\mathrm{psc}}(Z, \partial Z)$ :

$$
\mathcal{R}^{\mathrm{psc}}(Z, \partial Z)_{g_{0}, g_{1}}:=\left\{\bar{g} \in \mathcal{R}^{\mathrm{psc}}(Z, \partial Z) \mid c_{i}^{*} \bar{g}=g_{i}+\mathrm{d} t^{2} \text { on } Y_{i} \times[0,1], i=0,1\right\} .
$$

We note that each metric $\bar{g} \in \mathcal{R}^{\mathrm{psc}}(Z, \partial Z)_{g_{0}, g_{1}}$ provides a psc-bordism $(Z, \bar{g}):\left(Y_{0}, g_{0}\right) \rightsquigarrow\left(Y_{1}, g_{1}\right)$. We next assume $X$ is a manifold whose boundary $\partial X=Y_{0}$ is equipped with the metric $g_{0}$. Furthermore, we assume that both spaces $\mathcal{R}^{\mathrm{psc}}(X, \partial X)_{g_{0}}$ and $\mathcal{R}^{\mathrm{psc}}(Z, \partial Z)_{g_{0}, g_{1}}$ are non-empty. Now, by making use of the relevant collars, we glue together $X$ and $Z$ to obtain a smooth manifold which we denote $X \cup Z$ and which has boundary $\partial(X \cup Z)=Y_{1}$; see Figure 2 . 
In particular, we obtain the space $\mathcal{R}^{\mathrm{psc}}\left(X \cup Z, Y_{1}\right)_{g_{1}}$ of psc-metrics which restrict as $g_{1}+\mathrm{d} t^{2}$ on $c_{1}\left(Y_{1} \times[0,1]\right) \subset Z \subset X \cup Z$. Then for any metric $\bar{g} \in \mathcal{R}^{\mathrm{psc}}(Z, \partial Z)_{g_{0}, g_{1}}$, we obtain a map:

$$
\begin{aligned}
\mu_{Z, \bar{g}}: \mathcal{R}^{\mathrm{psc}}(X, \partial X)_{g_{0}} & \longrightarrow \mathcal{R}^{\mathrm{psc}}\left(X \cup Z, Y_{1}\right)_{g_{1}}, \\
h & \longmapsto h \cup \bar{g},
\end{aligned}
$$

where $h \cup \bar{g}$ is the metric obtained on $X \cup Z$ by the obvious gluing depicted in Figure 2 .

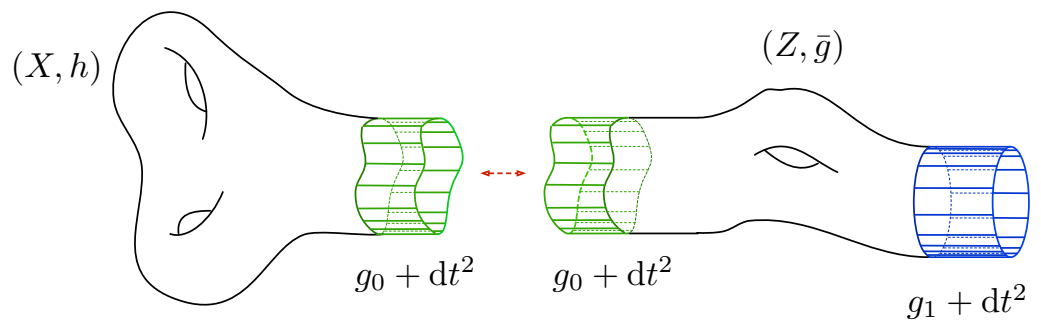

Figure 2. Attaching $(X, h)$ to $(Z, \bar{g})$ along a common boundary $\partial X=Y_{0}$.

Consider the case when the bordism $Z: Y_{0} \rightsquigarrow Y_{1}$ is an elementary bordism, i.e., when $Z$ is the trace of a surgery on $Y_{0}$ with respect to an embedding $\phi: S^{p} \times D^{q+1} \rightarrow Y_{0}$ with $p+q+1=$ $n-1=\operatorname{dim} Y_{0}$. Then we have the following.

Lemma 2.1 (surgery lemma; see $[8,9,18]$ ). Let $g_{0} \in \mathcal{R}^{+}\left(Y_{0}\right)$ be any metric. Assume $q \geq 2$. Then there exist metrics $g_{1} \in \mathcal{R}^{+}\left(Y_{1}\right)$ and $\bar{g} \in \mathcal{R}^{+}(Z, \partial Z)_{g_{0}, g_{1}}$ such that $(Z, \bar{g}):\left(Y_{0}, g_{0}\right) \rightsquigarrow$ $\left(Y_{1}, g_{1}\right)$ is a psc-bordism.

Such a bordism is usually called a Gromov-Lawson bordism (or GL-bordism for short). Here is a reformulation of the main technical result from [20]:

Theorem 2.2. Let $Z: Y_{0} \rightsquigarrow Y_{1}$ be an elementary bordism as above with $p, q \geq 2$. Then for any metric $g_{0} \in \mathcal{R}^{+}\left(Y_{0}\right)$ there exist metrics $g_{1} \in \mathcal{R}^{+}\left(Y_{1}\right)$ and $\bar{g} \in \mathcal{R}^{+}(Z, \partial Z)_{g_{0}, g_{1}}$ such that the map

$$
\mu_{Z, \bar{g}}: \mathcal{R}^{\mathrm{psc}}(X, \partial X)_{g_{0}} \stackrel{\simeq}{\rightarrow} \mathcal{R}^{\mathrm{psc}}\left(X \cup Z, Y_{1}\right)_{g_{1}}
$$

defined by (2.1), is a weak homotopy equivalence.

\subsection{Lifting to a Riemannian submersion}

An important aspect of our work involves lifting psc-metrics from the base of a smooth fibre bundle to a psc-submersion metric on its total space. In this section we briefly recall some pertinent facts and establish some notation.

Let $p: M \rightarrow B$, be the associated smooth fibre bundle to a principal $G$-bundle with respect to a Lie group, $G$, a compact smooth base manifold $B$ and compact smooth fibre manifold $F$, on which $G$ acts. Thus, the bundle has a structure map $f: B \rightarrow B G$. We denote by $\mathcal{H D}(p)$ the space of all horizontal distributions. This space is topologised in the obvious way as a subspace of smooth sections of the Grassmann bundle obtained by replacing each tangent space to $M$ with the Grassmannian of $\operatorname{dim} B$-dimensional subspaces. The following fact is elementary.

Proposition 2.3. The space $\mathcal{H} \mathcal{D}(p)$ is convex.

Suppose we have a $G$-invariant metric on $F$, denoted $g_{F}$, a metric on $B$ denoted $g_{B}$ and a horizontal distribution $\mathcal{H} \in \mathcal{H D}(p)$. By a well-known construction [2, Proposition 9.59], the triple, $\left(g_{B}, g_{F}, \mathcal{H}\right)$, determines a unique Riemannian submersion metric (with totally geodesic 
fibre metrics isometric to $g_{F}$ ) on the total space $M$. For simplicity, we fix the $G$-invariant metric on $F, g_{F}$, and consider constant multiples of this metric, $\tau g_{F}$, for some $\tau \in(0, \infty)$. This ability to scale up or down the fibre metric will give us important flexibility later on. We consider only the case when the fibre metric $g_{F}$ has non-negative scalar curvature and the base metric on $B$ has positive scalar curvature. Thus, we assume $\mathcal{R}^{\mathrm{psc}}(B) \neq \varnothing$.

We notice that the construction of a submersion metric varies continuously with respect to the triple $\left(g_{B}, \tau g_{F}, \mathcal{H}\right)$ and so we obtain a map

$$
\mathcal{S}: \mathcal{R}^{\mathrm{psc}}(B) \times \mathcal{H} \mathcal{D}(p) \times(0, \infty) \longrightarrow \mathcal{R}(M),
$$

where $\mathcal{S}(h, \mathcal{H}, \tau)$ is the unique submersion metric with base $(B, h)$, fibre $\left(F, \tau g_{F}\right)$ and horizontal distribution $\mathcal{H}$.

We denote by $\mathcal{R}_{\mathcal{S}}(M)$, the image of the map, $\mathcal{S}$, in $\mathcal{R}(M)$ and by $\mathcal{R}_{\mathcal{S}}^{\mathrm{psc}}(M)$ its subspace of psc-metrics. Thus, each element of $\mathcal{R}_{\mathcal{S}}^{\mathrm{psc}}(M)$ is a psc-submersion metric with respect to some base metric $g_{B} \in \mathcal{R}^{\mathrm{psc}}(B)$, some fibre metric $\tau g_{F}$ on $F$ (for some scaling constant $\tau>0$ ) and some horizontal distribution $\mathcal{H} \in \mathcal{H} \mathcal{D}(p)$. There are obvious maps from the space $\mathcal{R}_{\mathcal{S}}^{\text {psc }}(M)$ to the spaces $\mathcal{H} \mathcal{D}(p),(0, \infty)$ and $\mathcal{R}^{\mathrm{psc}}(B)$, sending a submersion metric to its respective horizontal distribution, fibre scaling constant and base metric. We consider the latter, denoting it

$$
\mathcal{B}: \mathcal{R}_{\mathcal{S}}^{\mathrm{psc}}(M) \rightarrow \mathcal{R}^{\mathrm{psc}}(B) .
$$

Thus, for any psc-submersion $\bar{g} \in \mathcal{R}_{\mathcal{S}}^{\mathrm{psc}}, \mathcal{B}(\bar{g})$ is the base metric on $B$. Finally, for any base metric $h \in \mathcal{R}^{\mathrm{psc}}(B)$, we denote by $\mathcal{R}_{\mathcal{S}}^{\mathrm{psc}}(M)_{h}$, the pre-image $\mathcal{B}^{-1}(h)$.

We will be interested in constructing a "lifting" map

$$
\mathcal{R}^{\mathrm{psc}}(B) \rightarrow \mathcal{R}^{\mathrm{psc}}(M)
$$

which sends each psc-metric on $B$, to a submersion metric on the the total space of the bundle $p: M \rightarrow B$. This will require some preliminary work.

From [2, Proposition 9.70] we know that the scalar curvature of such a metric, $\mathcal{S}(h, \mathcal{H}, \tau)$, denoted $s_{M}$, is given by the following formula of O'Neill:

$$
s_{M}=s_{h} \circ p+\frac{1}{\tau} s_{F}-\tau|A|^{2},
$$

where $s_{h}$ and $s_{F}$ are the scalar curvatures of the metrics $h \in \mathcal{R}^{\mathrm{psc}}(B)$ and $g_{F}$, and $A$ is the well-known tensor which is the obstruction to the integrability of the distribution. Note that the other well-known terms in this formula, in particular the tensor, $T$, vanish since the fibres are totally geodesic [2, Theorem 9.59]. By assumption, the sum of the first two terms in this formula is positive and so in order to ensure that $s>0$ we need to minimise the effect of $A$.

This formula varies continuously with respect to the choices of $h$ and $\mathcal{H}$; recall $g_{F}$ is fixed. In particular, we write $A:=A_{h, \mathcal{H}}$. Thus, by the compactness of $M$, there are continuous real valued parameters

$$
\mathrm{m}(h)=\min \left\{s_{h} \circ p(x): x \in M\right\} \quad \text { and } \quad \mathrm{M}_{A}(h, \mathcal{H})=\max \left\{\left|A_{h, \mathcal{H}}(x)\right|: x \in M\right\} .
$$

It follows that the scalar curvature, $s$, of the metric $\mathcal{S}(h, \mathcal{H}, \tau)$ satisfies

$$
s \geq \mathrm{m}(h)+\frac{1}{\tau} s_{L}-\tau \mathrm{M}_{A}(h, \mathcal{H})^{2} .
$$

To ensure positivity of $s$, we define $\bar{\tau}(h, \mathcal{H})$ by

$$
\bar{\tau}(h, \mathcal{H}):=\frac{\mathrm{m}(h)}{2 \mathrm{M}_{A}(h, \mathcal{H})^{2}} .
$$


Thus, we obtain a map

$$
\begin{aligned}
\mathcal{S}^{\mathrm{psc}}: \mathcal{R}^{\mathrm{psc}}(B) \times \mathcal{H} \mathcal{D}(p) & \rightarrow \mathcal{R}_{\mathcal{S}}^{\mathrm{psc}}(M), \\
(h, \mathcal{H}) & \mapsto \mathcal{S}(h, \mathcal{H}, \bar{\tau}(h, \mathcal{H})) .
\end{aligned}
$$

By fixing some horizontal distribution, $\mathcal{H}$, we define the map

$$
\mathcal{S}_{\mathcal{H}}^{\mathrm{psc}}: \mathcal{R}^{\mathrm{psc}}(B) \rightarrow \mathcal{R}_{\mathcal{S}}^{\mathrm{psc}}(M),
$$

by $\mathcal{S}_{\mathcal{H}}^{\mathrm{psc}}(h)=\mathcal{S}^{\mathrm{psc}}(h, \mathcal{H})$.

We now deduce some facts about this construction concerning homotopy. As an immediate consequence of the convexity of $\mathcal{H D}(p)$, we obtain the following.

Proposition 2.4. For any horizontal distributions, $\mathcal{H}, \mathcal{H}^{\prime} \in \mathcal{H} \mathcal{D}(p)$, the maps $\mathcal{S}_{\mathcal{H}}^{\mathrm{psc}}$ and $\mathcal{S}_{\mathcal{H}^{\prime}}^{\mathrm{psc}}$ are homotopy equivalent.

The lemma below will play an important role later on.

Lemma 2.5. The maps $\mathcal{B}$ and $\mathcal{S}_{\mathcal{H}}^{\mathrm{psc}}$ are homotopy inverse, thus determining a homotopy equivalence:

$$
\mathcal{R}^{\mathrm{psc}}(B) \simeq \mathcal{R}_{\mathcal{S}}^{\mathrm{psc}}(M)
$$

Proof. The composition $\mathcal{B} \circ \mathcal{S}_{\mathcal{H}}^{\text {psc }}$ is precisely the identity map on $\mathcal{R}^{\mathrm{psc}}(B)$. Consider now an arbitrary element $\bar{g} \in \mathcal{R}_{\mathcal{S}}^{\mathrm{psc}}(M)$. This element is uniquely represented as a triple $\bar{g}=\left(\bar{g}_{B}, \mathcal{H}_{\bar{g}}, \tau_{\bar{g}}\right)$, consisting of a base metric $\bar{g}_{B}=\mathcal{B}(\bar{g})$, a horizontal distribution and a scaling constant. Now,

$$
\mathcal{S}_{\mathcal{H}}^{\mathrm{psc}} \circ \mathcal{B}(\bar{g})=\mathcal{S}\left(\bar{g}_{B}, \mathcal{H}, \bar{\tau}\left(\bar{g}_{B}, \mathcal{H}\right)\right) .
$$

Thus, the desired homotopy is given by

$$
H_{t}(\bar{g}):=\mathcal{S}\left(\bar{g}_{B},(1-t) \mathcal{H}+t \mathcal{H}_{\bar{g}}, \bar{\tau}\left(\bar{g}_{B},(1-t) \mathcal{H}+t \mathcal{H}_{\bar{g}}\right)\right),
$$

where $t \in[0,1]$. That the resulting submersion metric $H_{t}(\bar{g})$ has positive scalar curvature for all $t$ is guaranteed by the construction of the function $\bar{\tau}$.

We now consider a variation of the scenario above which will be of great importance for us. We replace the bundle $p: M \rightarrow B$, with a smooth fibre bundle $\bar{p}: \bar{E} \rightarrow \bar{B}$. In this case, the base $\bar{B}$ and total space are smooth compact bordisms of manifolds, denoted $\bar{B}: B_{0} \rightsquigarrow B_{1}$ and $\bar{E}: E_{0} \rightsquigarrow E_{1}$, respectively. Here $E_{i}=\left.E\right|_{B_{i}}$ for $i \in\{0,1\}$. This bundle is also a $G$-bundle with fibre $L$, as above, and with structure map $\bar{f}: \bar{B} \rightarrow B G$ restricting to structure maps $f_{i}: B_{i} \rightarrow B G$ of the corresponding boundary bundles $p_{i}=\left.\bar{p}\right|_{E_{i}}: E_{i} \rightarrow B_{i}$. These boundary bundles are of exactly the type we discussed earlier with the role of $M$ played by $E_{i}$. We equip the bordism $\bar{B}$ with collars $c_{i}: B_{i} \times[0,2) \rightarrow \bar{B}, i=0,1$, along the boundary $\partial \bar{B}$. We will now introduce the following simplifying notation:

$$
\begin{aligned}
& \bar{B}_{c_{i}}:=c_{i}\left(B_{0} \times[0,1]\right), \quad \bar{B}_{c_{i}}^{\prime}:=\bar{B} \backslash \bar{B}_{c_{i}}, \\
& \bar{E}_{c_{i}}:=\left.\bar{E}\right|_{\bar{B}_{c_{i}}} \quad \text { and } \quad \bar{E}_{c_{i}}^{\prime}:=\bar{E} \backslash \bar{E}_{c_{i}}, \quad \text { where } \quad i \in\{0,1\} .
\end{aligned}
$$

As usual, we assume that $L$ is equipped with a fixed metric $g_{L}$ of non-negative scalar curvature. Suppose that $h_{0}$ and $h_{1}$ are a pair of psc-metrics on $B_{0}$ and $B_{1}$ and that $\bar{h}$ is a psc-metric in the (assumed to be non-empty) space of psc-bordism metrics $\mathcal{R}^{\mathrm{psc}}(\bar{B}, \partial \bar{B})_{h_{0}, h_{1}}$. Given some horizontal distribution $\overline{\mathcal{H}}$ on the bundle $\bar{p}$, the construction above allows us to "lift" the pscmetric $\bar{h}$ to psc-submersion metrics on the total space $\bar{E}$. In particular, to ensure our lifted metric has a product structure near the boundary of $\bar{E}$, we can impose the following admissibility condition on horizontal distributions. 
Definition 2.6. A horizontal distribution, $\overline{\mathcal{H}}$ on the bundle $\bar{p}: \bar{E} \rightarrow \bar{B}$ is said to be admissible provided it takes the form of a product $\mathcal{H}_{i} \times \mathbb{R}$ on the restrictions $\bar{E}_{c_{i}} \rightarrow \bar{B}_{c_{i}}$ for each $i \in\{0,1\}$.

We denote by $\mathcal{H} \mathcal{D}_{\text {adm }}(\bar{p})$, the subspace of admissible horizontal distributions on $\bar{p}$. That this space is convex follows from an elementary exercise.

For our purpose, we need to do something slightly more delicate. Let us assume that the boundary component $E_{0}$ of the total space is already equipped with a psc-metric $g_{0}$, one determining a Riemannian submersion of positive scalar curvature, $p_{0}:\left(E_{0}, g_{0}\right) \rightarrow\left(B_{0}, h_{0}\right)$, with respect to the fibre $\left(L, \tau_{0} g_{L}\right)$ for some constant $\tau_{0}>0$, and horizontal distribution $\mathcal{H}_{0} \in \mathcal{H D}\left(p_{0}\right)$. Thus, $g_{0} \in \mathcal{R}_{\mathcal{S}}^{\mathrm{psc}}\left(E_{0}\right)$ and more specifically lies in the image of the map

$$
S_{\mathcal{H}_{0}}^{\mathrm{psc}}: \mathcal{R}^{\mathrm{psc}}\left(B_{0}\right) \rightarrow \mathcal{R}_{\mathcal{S}}^{\mathrm{psc}}\left(E_{0}\right) .
$$

We wish to extend the psc-metric $g_{0}$ to a certain psc-metric on the total space $\bar{E}$. This is done in the following lemma.

Lemma 2.7. Let $\bar{p}: \bar{E} \rightarrow \bar{B}$ denote the bundle described above with structure map $\bar{f}: \bar{B} \rightarrow B G$ and fibre $L$, equipped with a fixed metric $g_{L}$ of non-negative scalar curvature. Let

$$
p_{0}=\left.\bar{p}\right|_{E_{0}}:\left(E_{0}, g_{0}\right) \rightarrow\left(B_{0}, h_{0}\right)
$$

be a psc-Riemannian submersion with respect to the fibre $\left(L, \tau_{0} g_{L}\right)$ for some constant $\tau_{0}>0$, and horizontal distribution $\mathcal{H}_{0} \in \mathcal{H} \mathcal{D}\left(p_{0}\right)$. For compact spaces $K_{1}$ and $K_{2}$, let $\bar{h}(x) \in \mathcal{R}^{\mathrm{psc}}(\bar{B}, \partial \bar{B})$, be a continuous family of psc-bordism metrics on $\bar{B}$, indexed by $x \in K_{1}$, and let $\overline{\mathcal{H}}(y) \in$ $\mathcal{H} \mathcal{D}_{\mathrm{adm}}(\bar{p})$ be a continuous family of admissible horizontal distributions indexed by $y \in K_{2}$. We further assume that, for all $x \in K_{1}$ and $y \in K_{2},\left.\bar{h}(x)\right|_{B_{0}}=h_{0}$ and $\left.\overline{\mathcal{H}}(y)\right|_{E_{0}}=\mathcal{H}_{0}$.

Then there is a constant $\bar{\tau}_{\min }>0$ and a corresponding family of psc-metrics $\bar{g}(x, y),(x, y) \in$ $K_{1} \times K_{2}$ on $\bar{E}$ which satisfies the following conditions.

(i) $\bar{p}:(\bar{E}, \bar{g}(x, y)) \rightarrow(\bar{B}, \bar{h}(x))$ is a Riemannian submersion with respect to the horizontal distribution $\overline{\mathcal{H}}(y)$.

(ii) Each psc-metric $\bar{g}(x, y)$ takes the form of a product $g_{i}(x, y)+\mathrm{d} t^{2}$ near the boundary components $E_{i}$, and satisfies $g_{0}(x, y)=g_{0}$ on $E_{0}$.

(iii) The Riemannian submersion obtained by restriction to $E_{1}$,

$$
p_{1}=\left.\bar{p}\right|_{E_{1}}:\left(E_{1}, g_{1}(x, y):=\left.\bar{g}(x, y)\right|_{E_{1}}\right) \rightarrow\left(B_{1}, h_{1}(x):=\left.\bar{h}(x)\right|_{B_{1}}\right),
$$

has fibre $\left(L, \bar{\tau}_{\min } g_{L}\right)$.

Proof. We begin by specifying $\bar{g}=\bar{g}(x, y)$ as, for all $(x, y)$, the product $g_{0}+\mathrm{d} t^{2}$ near the boundary component $E_{0}$. More precisely, $\bar{g}:=g_{0}+\mathrm{d} t^{2}$ on $\bar{E}_{c_{0}} \cong E_{0} \times[0,1]$. Thus, near $E_{0}$, the metric $\bar{g}$ forms part of a submersion $p_{0} \times \mathrm{Id}:\left(E_{0} \times I, g_{0}+\mathrm{d} t^{2}\right) \rightarrow\left(B_{0} \times I, h_{0}+\mathrm{d} t^{2}\right)$ with fibre $\left(L, \tau_{0} g_{L}\right)$ and horizontal distribution $\left.\overline{\mathcal{H}}\right|_{\bar{E}_{c_{0}}}=\mathcal{H}_{0} \times \mathbb{R}$.

Our next task is to extend this metric as a psc-submersion over the rest of $\bar{E}$. To maintain positive scalar curvature it may be necessary to continuously rescale the fibre metric, but in such a way as to preserve the original fibre metric near $E_{0}$. To simplify things, we make adjustments to the fibre metric only in $\bar{E}_{c_{0}}$ (and away from $E_{0}$ ), where the metric, $\bar{g}$, takes the form of a product $\bar{g}=g_{0}+\mathrm{d} t^{2}$. Consider, for constants $\tau>0$ and $b_{\tau} \geq 2$, smooth monotonic functions $\gamma_{\tau}:\left[0, b_{\tau}\right] \rightarrow[0, \tau]$ satisfying

(i) $\gamma_{\tau}(t)=\tau_{0}$ when $t \in[0,1]$,

(ii) $\gamma_{\tau}(t)=\tau$ when $t \in\left[b_{\tau}-1, b_{\tau}\right]$. 
We define $g_{0}(t)$ to be the submersion metric obtained on $E_{0} \times\{t\} \rightarrow B_{0} \times\{t\}$ with base metric $h_{0}$ and horizontal distribution $\mathcal{H}_{0}$ as before but replacing the fibre metric $\tau_{0} g_{L}$ with $\gamma_{\tau}(t) g_{L}$. Now replace the submersion $p_{0} \times \mathrm{Id}:\left(\bar{E}_{c_{0}}=E_{0} \times I, g_{0}+\mathrm{d} t^{2}\right) \rightarrow\left(B_{0} \times I, h_{0}+\mathrm{d} t^{2}\right)$, with $p_{0} \times \mathrm{Id}:\left(E_{0} \times\right.$ $\left.\left[0, b_{\tau}\right], g\left(\gamma_{\tau}(t)\right)+\mathrm{d} t^{2}\right) \rightarrow\left(B_{0} \times\left[0, b_{\tau}\right], h_{0}+\mathrm{d} t^{2}\right)$. The following proposition is an easy consequence of a well-known rescaling technique; see for example [12, Lemma 3] or [17, Lemma 1.3].

Proposition 2.8. For any constant $\tau>0$, the smooth function $\gamma_{\tau}$ above can be chosen so that the metric $g\left(\gamma_{\tau}(t)\right)+\mathrm{d} t^{2}$ has positive scalar curvature.

We will specify the choice of $\tau>0$ shortly. In the mean time, let us assume that $\gamma_{\tau}$ has been chosen to guarantee positive scalar curvature. Note that near $t=0$, the metric remains unchanged.

Consider now the other end of the cylinder, where $t=\tau_{b}$. Here we have a product of pscsubmersion metrics differing only from that at the $t=0$ end in that the fibre metric is now $\tau g_{L}$. No change has been made to the base metric and it is assumed to extend over the base bor$\operatorname{dism} \bar{B}$ as a psc-metric $\bar{h}$ which takes the form of a product metric $h_{1}+\mathrm{d} t^{2}$ near the boundary component $B_{1}$. For each $y$, the admissible horizontal distribution $\overline{\mathcal{H}}(y)$ extends $\mathcal{H}_{0} \times \mathbb{R}$ over the rest of $\bar{E}$, inducing, outside of the collar $\bar{E}_{c_{0}}$, Riemannian submersion metrics with base metric $\left.\bar{h}\right|_{\bar{B}_{c_{0}}^{\prime}}(x)$ and fibre metric $\tau g_{L}$. We need to ensure positive scalar curvature by choosing appropriately small $\tau$.

Suppose for any $(x, y)$, we choose $\tau=\bar{\tau}(\bar{h}(x), \overline{\mathcal{H}}(y))>0$, as defined in (2.2). This ensures, via the canonical variation formula [2, Proposition 9.70], that the submersion metric

$$
\mathcal{S}(\bar{h}(x), \overline{\mathcal{H}}(y), \bar{\tau}(\bar{h}(x), \overline{\mathcal{H}}(y)))
$$

on the bundle $\bar{E}_{c_{0}}^{\prime} \rightarrow \bar{B}_{c_{0}}^{\prime}$ with base metric $\left.\bar{h}(x)\right|_{\bar{B}_{c_{0}^{\prime}}}$, fibre metric $\bar{\tau}(\bar{h}(x), \overline{\mathcal{H}}(y)) g_{L}$ and horizontal distribution $\left.\overline{\mathcal{H}}(y)\right|_{\bar{E}_{c_{0}}^{\prime}}$ has positive scalar curvature. Thus, we set

$$
\tau=\bar{\tau}_{\min }:=\min \left\{\bar{\tau}(\bar{h}(x), \overline{\mathcal{H}}(y)):(x, y) \in K_{1} \times K_{2}\right\},
$$

to obtain a single scaling factor which works for all $(x, y)$. Finally, the metric $\bar{g}(x, y)$ is defined to be the submersion metric determined by the base $(\bar{B}, \bar{h}(x))$, fibre $L$ with varying metric $\gamma_{\bar{\tau}_{\text {min }}}(t) g_{L}$ on $E_{c_{0}}$ and $\bar{\tau}_{\text {min }} g_{L}$ on the rest of $\bar{E}$, as well as horizontal distribution $\overline{\mathcal{H}}(y)$.

\subsection{The case of manifolds with fibred singularities}

We now return to the manifolds with fibred singularities introduced in Section 1. Recall that $X$ is assumed to be a smooth manifold with boundary $\partial X \neq \varnothing$, which is the total space of a smooth bundle $\partial X \rightarrow \beta X$ with the fibre $L$ and structure map $f: \beta X \rightarrow B G$. Here $G$ is a subgroup of the isometry group of $g_{L}$, a Riemannian metric on $L$. Usually the Riemannian manifold $\left(L, g_{L}\right)$ is referred to as the link.

As explained in Section 1, we obtain a manifold with fibred singularities, $X_{\Sigma}$, given as $X_{\Sigma}:=$ $X \cup_{\partial X}-N(\beta X)$, by replacing the fibre $L$ with it's cone, $C(L)$, to obtain bundle $N(\beta X) \rightarrow \beta X$. Such a manifold a manifold is referred to as an $(L, G)$-manifold. For our constructions, we will require some further constraints on the link $\left(L, g_{L}\right)$, characterised by the following definition.

Definition 2.9. A link $\left(L, g_{L}\right)$ is simple if it satisfies one of the following conditions:

(a) $\left(L, g_{L}\right)$ is homogeneous with scalar curvature, $s_{g_{L}}$, a positive constant,

(b) $\left(L, g_{L}\right)=\left(S^{1}, \mathrm{~d} \theta^{2}\right)$,

(c) $L=\mathbb{Z}_{k}$. 
Henceforth, we assume that $\left(L, g_{L}\right)$ is a simple link. Before going forward with our constructions, we should clarify why the condition that the scalar curvature $s_{g_{L}}$ is non-negative constant is important here. by

In the case $(a), s_{g_{L}}$ is a positive constant. We define the cone metric $g_{C(L)}$ on the cone, $C(L)$,

$$
g_{C(L)}=\mathrm{d} t^{2}+c_{L}^{-2} t^{2} g_{L},
$$

where $c_{L}=\sqrt{\frac{\ell(\ell-1)}{s_{g_{L}}}}$ and $\operatorname{dim} L=\ell$. This is a warped product metric on $(0,1] \times L$ away from the cone point (where $t=0$ ) and is scalar flat, as demonstrated in the appendix.

The case $(b)$, when $L=S^{1}$, has special features. As $\operatorname{dim} L=\ell=1$ here, the definition of the metric $g_{C(L)}$ above coincides with the more general cone metric construction detailed in the appendix for the case when $g_{L}$ is scalar flat. In particular, formula (6.2) in the appendix gives that the scalar curvature of the metric $g_{C\left(S^{1}\right)}$ is identically zero. Moreover, any smooth $S^{1}$-bundle $p: Y \rightarrow B$ admits a free $S^{1}$-action on the manifold $Y$ such that $B=Y / S^{1}$. Then, according to a result by Bérard-Bergery [1, Theorem C], a manifold $Y$ admits an $S^{1}$-equivariant psc-metric if and only if the orbit space, the manifold $B=Y / S^{1}$ admits a psc-metric.

In the case $(c) L=\mathbb{Z}_{k}$. Then the cone $C\left(\mathbb{Z}_{k}\right)$ has the standard Euclidian metric, and we do not make any further assumptions. In any case, we can assume that the cone metric $g_{C(L)}$ is always scalar-flat (outside of its vertex).

We return now to the manifold $X$, which forms part a pseudo-manifold with $(L, G)$-singularities, $X_{\Sigma}=X \cup_{\partial X}-N(\beta X)$. Recall that $\partial X$ forms part of $G$-bundle, $p: \partial X \rightarrow \beta X$ with $G$ a subgroup of the isometry group of a fixed metric $g_{L}$ on the fibre $L$. We denote by $f: \beta X \rightarrow B G$, a structure map for this bundle. Throughout, $\operatorname{dim} X=n, \operatorname{dim} L=\ell$ and we reiterate that the link $\left(L, g_{L}\right)$ is assumed to be simple.

Bearing in mind the notions described in Section 2.2, we have a map

$$
\mathcal{S}: \mathcal{R}^{\mathrm{psc}}(\beta X) \times \mathcal{H} \mathcal{D}(p) \times(0, \infty) \longrightarrow \mathcal{R}(\partial X)
$$

which sends any triple $(h, \mathcal{H}, \tau)$ to the unique submersion metric on $\partial X$ with base $(\beta X, h)$, fibre $\left(L, \tau g_{L}\right)$ and horizontal distribution $\mathcal{H}$. Recall that the image of $\mathcal{S}$ is denoted $\mathcal{R}_{\mathcal{S}}(\partial X)$ and we further denote by $\mathcal{R}_{\mathcal{S}}^{\text {psc }}(\partial X)$ its subspace of psc-metrics. Thus, elements of $\mathcal{R}_{\mathcal{S}}^{\text {psc }}(\partial X)$ are psc-submersion metrics on the total space $\partial X$ with respect to some base metric in $\mathcal{R}^{\mathrm{psc}}(\beta X)$ and fibre metric $\tau g_{L}$ for some $\tau>0$. Recall that, for any $h \in \mathcal{R}^{\mathrm{psc}}(\beta X)$, the space $\mathcal{R}_{\mathcal{S}}^{\mathrm{psc}}(\partial X)_{h}$ consists of all psc-submersion metrics in $\mathcal{R}_{\mathcal{S}}^{\text {psc }}(\partial X)$ with base metric $h$. Recall also that by specifying a parameter $\bar{\tau}(h, \mathcal{H})$, we obtained "lifting" maps

$$
\begin{aligned}
\mathcal{S}^{\mathrm{psc}}: \mathcal{R}^{\mathrm{psc}}(\beta X) \times \mathcal{H} \mathcal{D}(p) & \rightarrow \mathcal{R}_{\mathcal{S}}^{\mathrm{psc}}(\partial X), \\
(h, \mathcal{H}) & \mapsto \mathcal{S}(h, \mathcal{H}, \bar{\tau}(h, \mathcal{H})),
\end{aligned}
$$

and

$$
\mathcal{S}_{\mathcal{H}}^{\mathrm{psc}}: \mathcal{R}^{\mathrm{psc}}(\beta X) \rightarrow \mathcal{R}_{\mathcal{S}}^{\mathrm{psc}}(\partial X),
$$

defined $\mathcal{S}_{\mathcal{H}}^{\text {psc }}(h):=\mathcal{S}^{\text {psc }}(h, \mathcal{H})$, for some fixed horizontal distribution $\mathcal{H}$. In particular, we recall that the homotopy type of the map $\mathcal{S}_{\mathcal{H}}^{\text {psc }}$ is independent of the choice of $\mathcal{H}$ and, moreover, the map $\mathcal{S}_{\mathcal{H}}^{\text {psc }}$ forms one direction in a homotopy equivalence (Lemma 2.5 above); its homotopy inverse is the map, $\mathcal{B}: \mathcal{R}_{\mathcal{S}}^{\mathrm{psc}}(\partial X) \rightarrow \mathcal{R}^{\mathrm{psc}}(\beta X)$, sending submersions to their base metrics.

Remark 2.10. We emphasise that the fibre metric $g_{L}$ and structure map $f: \beta X \rightarrow B G$ are assumed to be fixed throughout. Thus, while the map $\mathcal{S}$ (along with $\mathcal{S}^{\text {psc }}$ and $\mathcal{S}_{\mathcal{H}}^{\text {psc }}$ ) depend of course on these objects, and could be denoted by something like $\mathcal{S}_{f, g_{L}}$, we will not feature them in the notation. 


\subsection{Defining well-adapted metrics}

Returning to the manifold $X$, we consider the space of all psc-metrics, $\mathcal{R}^{\mathrm{psc}}(X, \partial X)$, which take a product structure near the boundary. Consider now the restriction map

$$
\text { res: } \mathcal{R}^{\mathrm{psc}}(X, \partial X) \rightarrow \mathcal{R}^{\mathrm{psc}}(\partial X), \quad \text { res }:\left.g \mapsto g\right|_{\partial X} .
$$

This map is very important for us because of the following fact:

Theorem $2.11([8,9])$. The restriction map res: $\mathcal{R}^{\mathrm{psc}}(X, \partial X) \rightarrow \mathcal{R}^{\mathrm{psc}}(\partial X)$ is a Serre fibre bundle.

We will make significant use of this fact later on. For now however, we consider the pre-image of the space $\mathcal{R}_{\mathcal{S}}^{\mathrm{psc}}(\partial X)$.

Definition 2.12. The space

$$
\mathcal{R}_{\mathcal{S}}^{\mathrm{psc}}(X, \partial X):=\operatorname{res}^{-1}\left(\mathcal{R}_{\mathcal{S}}^{\mathrm{psc}}(\partial X)\right) \subset \mathcal{R}^{\mathrm{psc}}(X, \partial X)
$$

is called the space of well-adapted psc-metrics on $X$.

There is a further subspace of $\mathcal{R}_{\mathcal{S}}^{\mathrm{psc}}(X, \partial X)$ which we must introduce. Suppose $h \in \mathcal{R}^{\mathrm{psc}}(\beta X)$ is a psc-metric on the Bockstein manifold $\beta X$. Recall that $\mathcal{R}_{\mathcal{S}}^{\mathrm{psc}}(\partial X)_{h} \subset \mathcal{R}_{\mathcal{S}}^{\mathrm{psc}}(\partial X)$, denotes the pre-image, $\mathcal{B}^{-1}(h)$. We now denote by $\mathcal{R}_{\mathcal{S}}^{\text {psc }}(X, \partial X)_{h}$, the space defined by

$$
\mathcal{R}_{\mathcal{S}}^{\mathrm{psc}}(X, \partial X)_{h}:=\operatorname{res}^{-1}\left(\mathcal{R}_{\mathcal{S}}^{\mathrm{psc}}(\partial X)_{h}\right)=\operatorname{res}^{-1}\left(\mathcal{B}^{-1}(h)\right) .
$$

All of this extends naturally to a definition of well-adapted metric on the pseudo-manifold $X_{\Sigma}=X \cup_{\partial X}-N(\beta X)$. A well adapted psc-metric $g \in \mathcal{R}_{\mathcal{S}}^{\mathrm{psc}}(X, \partial X)$, has associated to it a unique Riemannian submersion $p:\left(\partial X, g^{\partial}\right) \rightarrow\left(\beta X, h^{\beta}\right)$ with a fibre $\left(L, \tau g_{L}\right)$ for some constant $\tau>0$. This determines

(i) a cone metric, $g_{C(L)}$, as defined in (2.3), although with $g_{L}$ now replaced by $\tau g_{L}$ in that formula,

(ii) and an attaching metric $g_{\text {att }(L)}$.

In turn, we obtain a submersion metric on $N(\beta X)$ obtained by equipping the fibres with $g_{\text {att }(L)} \cup$ $g_{C(L)}$. This metric then attaches to $g$ in the obvious way to yield a metric on $X_{\Sigma}$. This process can be thought of as a map

$$
i_{\Sigma}: \mathcal{R}_{\mathcal{S}}^{\mathrm{psc}}(X, \partial X) \longrightarrow \mathcal{R}\left(X_{\Sigma}\right) .
$$

This map is easily seen to be a homeomorphism onto its image. This leads to the following definition.

Definition 2.13. The image of the map $i_{\Sigma}$, denoted $\mathcal{R}^{\mathrm{psc}}\left(X_{\Sigma}\right)$, is called the space of well adapted psc-metrics on $X_{\Sigma}$.

This space, $\mathcal{R}^{\mathrm{psc}}\left(X_{\Sigma}\right)$, is the space we are most interested in studying.

Remark 2.14. It is worth emphasising that the space $\mathcal{R}_{\mathcal{S}}^{\mathrm{psc}}(\partial X)$ (as well the space $\mathcal{R}_{\mathcal{S}}^{\mathrm{psc}}(X, \partial X)$ ) could be empty even though the boundary $\partial X$ has a psc-metric. A simple example is the following. Let $\beta X=K 3$. Then for any prime generator $c \in H^{2}(K 3 ; \mathbb{Z})=\mathbb{Z}^{22}$ a corresponding map $c: K 3 \rightarrow \mathbb{C P}^{\infty}$ gives a bundle $Z \rightarrow \beta X$, where $Z$ is a simply-connected spin manifold which, of course, admits a psc-metric. Moreover, $Z$ is a spin boundary: $Z=\partial X$. However, no psc-metric on $Z$ could be invariant under $S^{1}$-action, otherwise, according to Bérard-Bergery [1, Theorem C], it would imply the existence of a psc-metric on $K 3$. 
As we mentioned above, there are two types of surgery that could be performed on $X_{\Sigma}$ : the first one on its resolution, the interior of $X$, and the second one on the structure map $f: \beta X \rightarrow B G$. We consider the latter. Moreover, for now it is convenient to cut the singularity out and to work with a smooth manifold $X$ whose boundary is fibred over $\beta X$ with fibre $L$. We use the notation $(X, \beta X, f)$ for such manifold, where the boundary $\partial X$ of $X$ is a total space of the fibre bundle from the diagram:

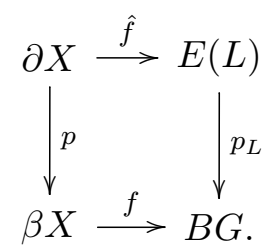

Here $p_{L}: E(L) \rightarrow B G$ is the universal fibre bundle with the fibre $L$ and the structure group $G$.

We recall the earlier bordism of smooth compact manifolds, $\bar{B}: B_{0} \rightsquigarrow B_{1}$. We assume now that this is an elementary bordism with $B_{0}=\beta X$ and, as before, $\bar{f}: \bar{B} \rightarrow B G$ a map such that $\left.\bar{f}\right|_{B_{0}}=f_{0}=f$. We will use the notation $(\bar{B}, \bar{f}):\left(B_{0}, f_{0}\right) \rightsquigarrow\left(B_{1}, f_{1}\right)$. Let $\bar{p}: \bar{E} \rightarrow \bar{B}$ be a corresponding fibre bundle with fibre $L$ and structure group $G$. By construction, $\left.\bar{E}\right|_{B_{0}}=$ $E_{0}=\partial X$, and $\left.\bar{p}\right|_{E_{0}}=p$. Then the manifold $\bar{E}$ gives a bordism $\bar{E}: E_{0} \rightsquigarrow E_{1}$, where $E_{1}=\left.E\right|_{B_{1}}$. As before, we assume that the bordism $\bar{B}$ is equipped with collars $c_{i}: B_{i} \times[0,2) \rightarrow \bar{B}, i=0,1$, along the boundary $\partial \bar{B}$.

We now equip $B_{0}=\beta X$ with the metric $h_{0}^{\beta}=h^{\beta}\left(=g_{\beta X}\right)$. We assume that there is a psc-

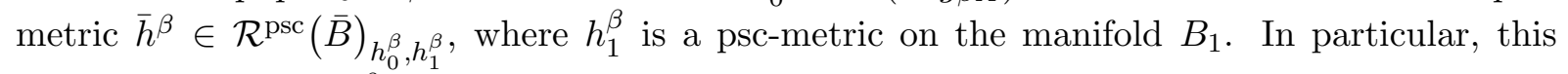
means that $c_{i}^{*} \bar{h}^{\beta}=h_{i}^{\beta}+\mathrm{d} t^{2}$, with respect to the collars $c_{i}: B_{i} \times[0,2) \hookrightarrow \bar{B}, i=0,1$ near the boundary $\partial \bar{B}=B_{0} \sqcup B_{1}$. Thus, the metric $\bar{h}^{\beta}$ provides a psc-bordism

$$
\left(\bar{B}, \bar{f}, \bar{h}^{\beta}\right):\left(B_{0}, f_{0}, h_{0}^{\beta}\right) \rightsquigarrow\left(B_{1}, f_{1}, h_{1}^{\beta}\right) .
$$

The structure map $\bar{f}: \bar{B} \rightarrow B G$ determines a bordism $\bar{E}: E_{0} \rightsquigarrow E_{1}$, where $\bar{E}$ is a pull-back of the universal $(L, G)$-fibration:

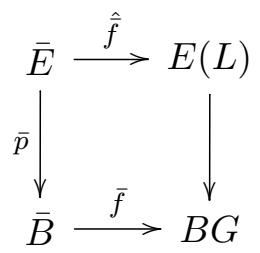

with $\left.\bar{E}\right|_{B_{0}}=E_{0}$ and $\left.\bar{E}\right|_{B_{1}}=E_{1}$. Now we glue the manifolds $X$ and $\bar{E}$ (again, making use of collars near their boundaries) to obtain the manifold $X_{1}=X \cup_{\partial X} \bar{E}$ with boundary $\partial X_{1}=E_{1}$, which is the total space of the $(L, G)$-fibration $p_{1}: \partial X_{1} \rightarrow B_{1}$. We denote $\beta X_{1}=B_{1}$. We denote by $\mathcal{S}_{1}$, the analogue of the map $\mathcal{S}$ in (2.4), for the bundle, $p_{1}: \partial X_{1} \rightarrow B_{1}$ and by $\mathcal{R}_{\mathcal{S}_{1}}^{\text {psc }}\left(X_{1}, \partial X_{1}\right)_{h_{1}^{\beta}}$, the analogous space of psc-metrics on $X_{1}$ with boundary metric a psc-submersion which restricts on the base as the psc-metric $h_{1}^{\beta}$. We will shortly employ the psc-submersion construction from Lemma 2.7 to obtain a map

$$
\mathcal{R}_{\mathcal{S}}^{\mathrm{psc}}(X, \partial X)_{h_{0}^{\beta}} \longrightarrow \mathcal{R}_{\mathcal{S}_{1}}^{\mathrm{psc}}\left(X_{1}, \partial X_{1}\right)_{h_{1}^{\beta}}
$$

an analogue of the map in (2.1). In preparation for this, there are some objects we must reintroduce.

In the preamble to Lemma 2.7, we considered spaces of horizontal distributions, $\mathcal{H} \mathcal{D}\left(p_{0}\right)$ and $\mathcal{H} \mathcal{D}_{\text {adm }}(\bar{p})$. In the latter case, we recall that distributions satisfy a product structure near 
the boundary components $E_{i}$. It will be useful for us to consider the space of maps from $\mathcal{H D}\left(p_{0}\right)$ to $\mathcal{H} \mathcal{D}_{\text {adm }}(\bar{p})$, which we denote $\mathcal{F}\left(\mathcal{H D}\left(p_{0}\right), \mathcal{H} \mathcal{D}_{\text {adm }}(\bar{p})\right)$, under the usual compact-open topology. In particular, we will be interested in maps $\xi: \mathcal{H D}\left(p_{0}\right) \rightarrow \mathcal{H} \mathcal{D}_{\text {adm }}(\bar{p})$ which satisfy the condition that $\left.\xi(\mathcal{H})\right|_{E_{0}}=\mathcal{H}$. Such maps are easy to construct. For example, fix a disribution $\overline{\mathcal{H}}$ on $\left.\bar{E}\right|_{\bar{B} \backslash c_{0}(B \times[0,1])}$ which takes a product structure, $\mathcal{H}_{1} \times \mathbb{R}$, on $\left.\bar{E}\right|_{c_{0}(B \times[1,2))}$. Then define $\xi(\mathcal{H})$ as $\mathcal{H} \times \mathbb{R}$ on $\left.\bar{E}\right|_{c_{0}(B \times[0,1]}, \overline{\mathcal{H}}$ on $\left.\bar{E}\right|_{\bar{B} \backslash c_{0}(B \times[0,2))}$ while continuously transitioning between $\mathcal{H}$ and $\mathcal{H}_{1}$ along $\left.E\right|_{c_{0}(B \times[1,2))}$. We denote by $\mathcal{F}_{0}\left(\mathcal{H} \mathcal{D}\left(p_{0}\right), \mathcal{H} \mathcal{D}_{\text {adm }}(\bar{p})\right)$, the subspace of $\mathcal{F}\left(\mathcal{H D}\left(p_{0}\right), \mathcal{H} \mathcal{D}_{\text {adm }}(\bar{p})\right)$, defined by

$$
\mathcal{F}_{0}\left(\mathcal{H D}\left(p_{0}\right), \mathcal{H} \mathcal{D}_{\mathrm{adm}}(\bar{p})\right):=\left\{\xi \in \mathcal{F}\left(\mathcal{H D}\left(p_{0}\right), \mathcal{H} \mathcal{D}_{\mathrm{adm}}(\bar{p})\right):\left.\xi(\mathcal{H})\right|_{E_{0}}=\mathcal{H}\right\}
$$

The proof of the following proposition is an elementary exercise.

Proposition 2.15. The spaces $\mathcal{F}\left(\mathcal{H D}\left(p_{0}\right), \mathcal{H} \mathcal{D}_{\mathrm{adm}}(\bar{p})\right)$ and $\mathcal{F}_{0}\left(\mathcal{H D}\left(p_{0}\right), \mathcal{H} \mathcal{D}_{\mathrm{adm}}(\bar{p})\right)$ are convex.

We next recall that a metric $g \in \mathcal{R}_{\mathcal{S}}^{\mathrm{psc}}(X, \partial X)_{h_{0}^{\beta}}$ restricts on the boundary $\partial X$ as a submersion metric, $g^{\partial}=\left.g\right|_{\partial X}$ over the base metric $h_{0}^{\beta}$, with fibre metric $\tau_{0}(g) g_{L}$ (for some fibre metric scaling constant $\tau_{0}(g)>0$ ) and horizontal distribution $\mathcal{H}_{0}(g)$. Both the fibre metric scaling constant and the horizontal distribution vary continuously with respect to $g$. We now choose a map $\xi: \mathcal{H D}(p) \longrightarrow \mathcal{H} \mathcal{D}_{\text {adm }}(\bar{p})$ from the space $\mathcal{F}_{0}\left(\mathcal{H D}\left(p_{0}\right), \mathcal{H} \mathcal{D}_{\text {adm }}(\bar{p})\right)$. Thus, for any $g \in$ $\mathcal{R}_{\mathcal{S}}^{\text {psc }}(X, \partial X)_{h_{0}^{\beta}}$, we obtain a distribution $\xi\left(\mathcal{H}_{0}(g)\right)$ on the total space $\bar{E}$, extending $\mathcal{H}_{0}(g)$ from $E_{0}$. This allows us to specify a map

$$
\begin{aligned}
\mu_{\left(\bar{B}, \bar{f}, \bar{h}^{\beta}\right)}: \mathcal{R}_{\mathcal{S}}^{\mathrm{psc}}(X, \partial X)_{h_{0}^{\beta}} & \longrightarrow \mathcal{R}_{\mathcal{S}_{1}}^{\mathrm{psc}}\left(X_{1}, \partial X_{1}\right)_{h_{1}^{\beta}}, \\
g & \longmapsto g \cup \bar{g}^{\partial},
\end{aligned}
$$

where $\bar{g}^{\partial}$ is the metric obtained in Lemma 2.7 above with respect to the triple of base met-

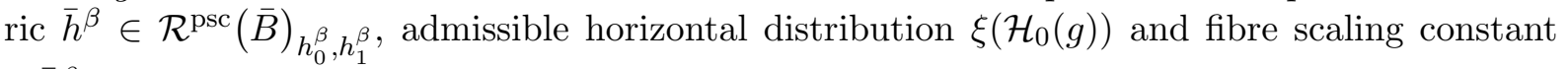
$\bar{\tau}\left(\bar{h}^{\beta}, \xi\left(\mathcal{H}_{0}(g)\right)\right.$ ) (as defined in (2.2)). The following is an easy consequence of Lemma 2.7 .

Corollary 2.16. The homotopy type of the map $\mu_{\left(\bar{B}, \bar{f}, \bar{h}^{\beta}\right)}$ is invariant of the psc-isotopy type of the base metric $\bar{h}^{\beta}$, as well as the choice of distribution map $\xi \in \mathcal{F}_{0}\left(\mathcal{H D}\left(p_{0}\right), \mathcal{H} \mathcal{D}_{\mathrm{adm}}(\bar{p})\right)$.

As our work concerns only homotopy type, we feel justified in suppressing the role of $\xi$, in the notation of the map (2.7) above.

Now we are ready to state our main technical result which is similar to Theorem 2.2.

Theorem 2.17. Let $(X, \beta X, f)$ be a manifold with fibred singularities, i.e., the boundary $\partial X$ is a total space of an $(L, G)$-fibration $\partial X \rightarrow \beta X$ given by the structure map $f: \beta X \rightarrow B G$, where $\operatorname{dim} X=n, \operatorname{dim} L=\ell$. Furthermore, we assume $(\bar{B}, \bar{f}):\left(B_{0}, f_{0}\right) \rightsquigarrow\left(B_{1}, f_{1}\right)$ is an elementary bordism with $p, q \geq 2$, where $B_{0}=\beta X$. Then for any psc-metric $h_{0}^{\beta}$ on $B_{0}$ there exist pscmetrics $h_{1}^{\beta}$ on $B_{1}$ and $\bar{h}^{\beta} \in \mathcal{R}^{\mathrm{psc}}(\bar{B})_{h_{0}^{\beta}, h_{1}^{\beta}}$ such that the map

$$
\begin{array}{cl}
\mu_{\left(\bar{B}, \bar{f}, \bar{h}^{\beta}\right)}: & \mathcal{R}_{\mathcal{S}}^{\mathrm{psc}}(X, \partial X)_{h_{0}^{\beta}} \longrightarrow \mathcal{R}_{\mathcal{S}_{1}}^{\mathrm{psc}}\left(X_{1}, \partial X_{1}\right)_{h_{1}^{\beta}}, \quad g \longmapsto g \cup \bar{g}^{\partial}, \\
& X_{1}=X \cup_{\partial X=E_{0}} \bar{E}, \quad \beta X_{1}=E_{1},
\end{array}
$$

defined as in (2.7) above, is a weak homotopy equivalence.

Remark 2.18. The construction of the metrics $h_{1}^{\beta}$ on $B_{1}$ and $\bar{h}^{\beta}$ on $\bar{B}$ used in this theorem, follows from surgery techniques pioneered by Gromov and Lawson [12], and later generalised by others. It should be pointed out however that, given such a metric, $h_{1}^{\beta}$, the theorem works equally well if $h_{1}^{\beta}$ is replaced by any psc-metric which is psc-isotopic to $h_{1}^{\beta}$, i.e., lies in the same path component of the space $\mathcal{R}^{\mathrm{psc}}\left(B_{1}\right)$. 


\section{Proof of Theorem 2.17}

\subsection{Some standard metric constructions}

Here we briefly a recall a couple of standard metric constructions. These constructions are discussed in detail in [19, Section 5].

We fix some constants $\delta>0$ and $\lambda \geq 0$. Then a $(\delta-\lambda)$-torpedo metric on the disk $D^{n}$, denoted $g_{\text {torp }}^{n}(\delta)_{\lambda}$, is a psc-metric which roughly takes the form a round hemisphere of radius $\delta>0$ near the centre before transitioning into a cylinder of radius $\delta$ and length $\lambda \geq 0$ near the boundary; see first image in Figure 3 below.

Letting $D_{+}^{n}$ denote the upper hemi-disk, we obtain the metric $g_{\text {torp }+}^{n}(\delta)_{\lambda}:=\left.g_{\text {torp }}^{n}(\delta)_{\lambda}\right|_{D_{+}^{n}}$; see second image in Figure 3. We call $g_{\text {torp }+}^{n}(\delta)_{\lambda}$ a half-torpedo metric. Let $\lambda_{2}>0$ be some constant. Next, we consider the cylinder $D^{n-1} \times\left[0, \lambda_{2}\right]$ equipped with the metric $g_{\text {torp }}^{n-1}(\delta)_{\lambda_{1}}+\mathrm{d} t^{2}$ and attach a half-disk $D_{+}^{n}$ with half-torpedo metric $g_{\text {torp }+}^{n}(\delta)_{\lambda_{1}}$ along $D^{n-1} \times\{0\}$. We denote the resulting Riemannian manifold by $\left(D_{\text {stretch }}^{n}, \hat{g}_{\text {torp }}^{n}(\delta)_{\lambda_{1}, \lambda_{2}}\right)$. This is depicted in the third image in Figure 3.

Typically, we will not care so much about the $\lambda_{2}$-parameter but only $\lambda_{1}$ which we regard as the vertical height of this metric. Moreover, we will usually be interested in the case when $\lambda_{1}=1$ and when $\delta=1$. With this in mind we make use of the following notational simplifications.

$$
\begin{aligned}
& g_{\text {torp }}^{n}:=g_{\text {torp }}^{n}(1)_{1} . \\
& g_{\text {torp }+}^{n}:=g_{\text {torp }+}^{n}(1)_{1} . \\
& \hat{g}_{\text {torp }}^{n}(\delta)_{\lambda_{1}}:=\hat{g}_{\text {torp }}^{n}(\delta)_{\lambda_{1}, \lambda_{2}}, \quad \text { where } \quad \lambda_{2} \quad \text { is arbitrary. } \\
& \hat{g}_{\text {torp }}^{n}:=\hat{g}_{\text {torp }}^{n}(1)_{1}=\hat{g}_{\text {torp }}^{n}(1)_{1,1} .
\end{aligned}
$$

The following proposition follows immediately from [19, Proposition 3.1.6].

Proposition 3.1. Let $n \geq 3, \delta>0$ and $\lambda, \lambda_{1}, \lambda_{2} \geq 0$.

(i) The metrics $g_{\mathrm{torp}}^{n}(\delta)_{\lambda}, g_{\mathrm{torp}+}^{n}(\delta)_{\lambda}$ and $\hat{g}_{\mathrm{torp}}^{n}(\delta)_{\lambda_{1}, \lambda_{2}}$ have positive scalar curvature.

(ii) For any constant $b \geq 0$ and any $\lambda, \lambda_{1}, \lambda_{2} \geq 0$, there exists $\delta>0$ so that the scalar curvature of the metrics $g_{\text {torp }}^{n}(\delta)_{\lambda}, g_{\text {torp }+}^{n}(\delta)_{\lambda}$ and $\hat{g}_{\text {torp }}^{n}(\delta)_{\lambda_{1}, \lambda_{2}}$ is bounded below by $b$.
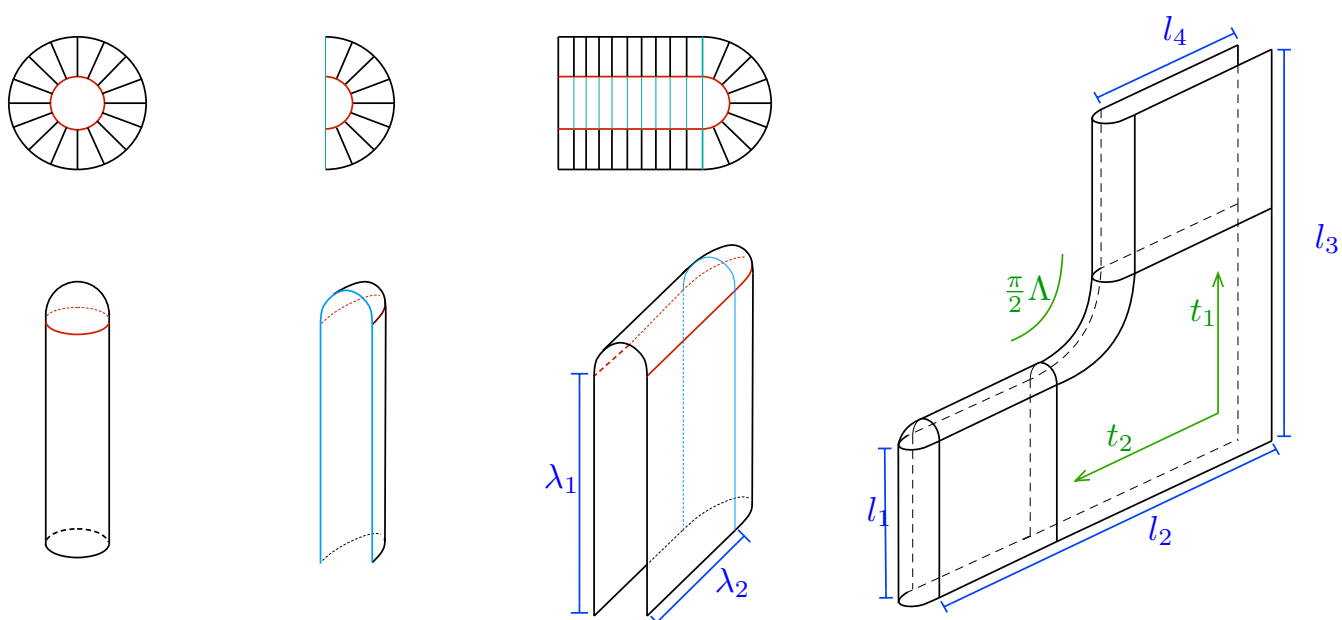

Figure 3. The metrics $g_{\text {torp }}^{n}(\delta)_{\lambda}, g_{\text {torp }+}^{n}(\delta)_{\lambda}$ and $\hat{g}_{\text {torp }}^{n}(\delta)_{\lambda_{1}, \lambda_{2}}$ (bottom) on the manifolds $D^{n}, D_{+}^{n}$ and $D_{\text {stretch }}^{n}$ (top) followed by the boot metric $g_{\text {boot }}^{n}(\delta)_{\Lambda, \bar{l}}$. 
We now consider product metrics $g_{\text {torp }}^{n-1}(\delta)_{\lambda}+\mathrm{d} t^{2}$ on the cylinder $D^{n-1} \times I$. It is shown in [19, Section 5], provided $n \geq 4$, that any such product metric $g_{\text {torp }}^{n-1}(\delta)_{\lambda}+\mathrm{d} t^{2}$ can be moved by isotopy through psc-metrics to a particular psc-metric called a $\delta$-boot metric. A detailed account of how such metrics are constructed can be found in [19]. Here we will provide brief description.

(i) Beginning with some torpedo metric, $g_{\mathrm{torp}}^{n-1}(\delta)_{\lambda}$, trace out a cylinder of torpedo metrics before bending the cylinder around an angle of $\frac{\pi}{2}$ to finish as a Riemannian cylinder (perpendicular to the first one) in the direction suggested by the rightmost image of Figure 3. The resulting object has two cylindrical ends, one of the form $\mathrm{d} t_{1}^{2}+g_{\text {torp }}^{n-1}(\delta)_{\lambda}$ and the other $\mathrm{d} t_{2}^{2}+g_{\text {torp }}^{n-1}(\delta)_{\lambda}$, where $t_{1}$ and $t_{2}$ are orthogonal coordinates depicted in Figure 3.

(ii) In order to ensure the resulting metric has positive scalar curvature, the bending is controlled by a paramater $\Lambda>0$. Essentially, the bending takes place along a quarter-circle of radius $\Lambda>0$. A sufficiently large choice of $\Lambda$ ensures that curvature arising from the bend is small and positivity of the scalar curvature arising from the torpedo factor dominates.

(iii) Away from the "caps" of the torpedos, this metric takes the form $\mathrm{d} t_{1}^{2}+\mathrm{d} t_{2}^{2}+\delta^{2} \mathrm{~d} s_{n-1}^{2}$. This part can easily be extended to incorporate the corner depicted in the rightmost image of Figure 3 and so that the necks of the torpedo "ends" have any desired lengths, $l_{1}$ and $l_{4}$ (determining along with $\Lambda$ the distances $l_{2}$ and $l_{3}$ ).

(iv) Finally, we smoothly "cap-off" the cylindrical end which takes the form $\mathrm{d} t_{1}^{2}+g_{\text {torp }}^{n-1}(\delta)_{l_{1}}$, by attaching a half-torpedo metric, $g_{\text {torp }+}^{n}(\delta)_{l_{1}}$. This is the so-called "toe" of the boot metric.

The resulting metric is denoted $g_{\text {boot }}^{n}(\delta)_{\Lambda, \bar{l}}$, where $\Lambda>0$ is the bending constant discussed above and $\bar{l}=\left(l_{1}, l_{2}, l_{3}, l_{4}\right) \in(0, \infty)^{4}$ determines the various neck-lengths. As we mentioned above, $\Lambda$ may need to be chosen to be large (although can always be found) and will depend on $\delta$. While the choices of $l_{1}$ and $l_{4}$ are arbitrary, the constants $l_{2}$ and $l_{3}$ are determined by $\Lambda, l_{1}$ and $l_{4}$.

\subsection{Back to the proof of Theorem 2.17}

The proof follows from that of [20, Theorem A]. We will provide a brief review of the main steps of that proof and show that it goes through perfectly well in our case. The strategy is to decompose the map

$$
\mu_{\left(\bar{B}, \bar{f}, \bar{h}^{\beta}\right)}: \mathcal{R}_{\mathcal{S}}^{\mathrm{psc}}(X, \partial X)_{h_{0}^{\beta}} \longrightarrow \mathcal{R}_{\mathcal{S}_{1}}^{\mathrm{psc}}\left(X_{1}, \partial X_{1}\right)_{h_{1}^{\beta}},
$$

into a composition of three maps as shown in the commutative diagram below:

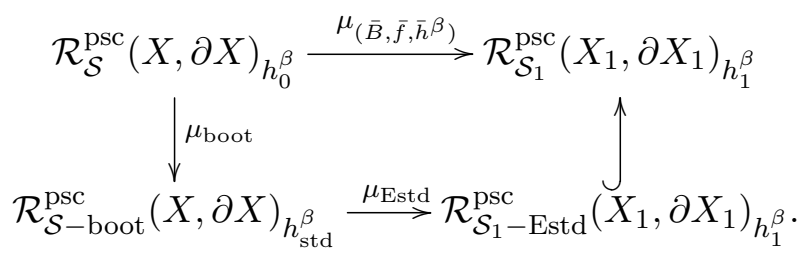

Here the right vertical map denotes inclusion. We will define the spaces $\mathcal{R}_{\mathcal{S} \text {-boot }}^{\mathrm{psc}}(X, \partial X)_{h_{\text {std }}^{\beta}}$ and $\mathcal{R}_{\mathcal{S}_{1} \text {-Estd }}^{\mathrm{psc}}\left(X_{1}, \partial X_{1}\right)_{h_{1}^{\beta}}$ and the maps $\mu_{\text {boot }}$ and $\mu_{\text {Estd }}$ in due course. The point is to show that each of these maps is a weak homotopy equivalence.

We denote by $k=n-\ell-1=\operatorname{dim} \beta X=B_{0}$. We consider carefully the elementary bordism $(\bar{B}, \bar{f}):\left(B_{0}, f_{0}\right) \rightsquigarrow\left(B_{1}, f_{1}\right)$. The manifold $\bar{B}$ is given by attaching a handle $D^{p+1} \times D^{q+1}$ to $B_{0}$ 
along the embeddings $\phi: S^{p} \times D^{q+1} \hookrightarrow B_{0}$, where $p+q+1=k, q \geq 2$. We would like to have some flexibility for the embedding $\phi$. We introduce the following family of rescaling maps:

$$
\begin{aligned}
\sigma_{\rho}: S^{p} \times D^{q+1} & \longrightarrow S^{p} \times D^{q+1}, \\
(x, y) & \longmapsto(x, \rho y),
\end{aligned}
$$

where $\rho \in(0,1]$. We set

$$
\phi_{\rho}:=\phi \circ \sigma_{\rho}: S^{p} \times D^{q+1} \hookrightarrow B_{0}
$$

and $N_{\rho}:=\phi_{\rho}\left(S^{p} \times D^{q+1}\right)$, abbreviating $N:=N_{1}$ and $\phi:=\phi_{1}$. Let $T_{\phi}$ be the trace of the surgery on $B_{0}$ with respect to $\phi$. We denote by $\mathcal{R}_{\text {std }}^{\mathrm{psc}}\left(B_{0}\right)$, the space defined as follows:

$$
\mathcal{R}_{\mathrm{std}}^{\mathrm{psc}}\left(B_{0}\right):=\left\{g \in \mathcal{R}^{\mathrm{psc}}\left(B_{0}\right): \phi_{\frac{1}{2}}^{*} g=\mathrm{d} s_{p}^{2}+g_{\text {torp }}^{q+1} \text { on } S^{p} \times D^{q+1}\right\} .
$$

According to Chernysh's theorem [8,9], the inclusion

$$
\mathcal{R}_{\text {std }}^{\mathrm{psc}}\left(B_{0}\right) \subset \mathcal{R}^{\mathrm{psc}}\left(B_{0}\right),
$$

is a weak homotopy equivalence. A major step in the proof of this theorem is the fact (which follows easily enough from the original Gromov-Lawson construction in [12]) that for any pscmetric $h^{\beta} \in \mathcal{R}^{\mathrm{psc}}\left(B_{0}\right)$, there is an isotopy $h_{t}^{\beta}, t \in I$ of metrics in $\mathcal{R}^{\mathrm{psc}}\left(B_{0}\right)$ connecting $h_{0}^{\beta}=h^{\beta}$ to a psc-metric $h_{\text {std }}^{\beta} \in \mathcal{R}_{\text {std }}^{\text {psc }}\left(B_{0}\right)$. By a well known argument (see [20, Lemma 2.3.2]), this isotopy gives rise to a concordance: $\bar{h}_{\text {con }}^{\beta}$ on $B_{0} \times[0, \lambda+2]$ for some $\lambda>0$ which takes the form of product metrics:

$$
h^{\beta}+\mathrm{d} t^{2} \quad \text { on } \quad B_{0} \times[\lambda+1, \lambda+2] \quad \text { and } \quad g_{\text {std }}+\mathrm{d} t^{2} \quad \text { on } \quad B_{0} \times[0,1] .
$$

Note that on the slice $N_{\frac{1}{2}} \times[0,1]$, the metric $\bar{h}_{\text {con }}^{\beta}$ pulls back to a metric of the form

$$
\mathrm{d} s_{p}^{2}+g_{\text {torp }}^{q+1}+\mathrm{d} t^{2} .
$$

Making use of [20, Lemma 5.2.5] we can perform an isotopy of the metric $\bar{h}_{\text {con }}^{\beta}$, adjusting only on $N_{\frac{1}{2}} \times[0,1]$, to replace the $g_{\text {torp }}^{q+1}+\mathrm{d} t^{2}$ factor in $(3.2)$ with $g_{\text {boot }}^{q+2}(1)_{\Lambda, \bar{l}}$ for some appropriately large $\Lambda>0$ and with $\bar{l}$ satisfying $l_{1}=l_{4}=1$. We denote the resulting psc-metric $\bar{h}_{\text {pre }}^{\beta}$ on $B_{0} \times$ $[0, \lambda+2]$. We consider $B_{0} \times[0, \lambda+2]$ as a long collar of $\bar{B}$ and assume that the map $\bar{f}$ restricted to $B_{0} \times[0, \lambda+2]$ is given by $\bar{f}(x, t)=f_{0}(x)$. Let $\bar{E}_{0}$ be a manifold given by pulling back the fibre bundle

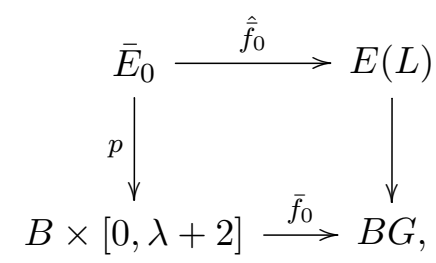

where $\bar{f}_{0}$ is a restriction of $\bar{f}$.

We now use the metric $\bar{h}_{\text {pre }}^{\beta}$ on $B_{0} \times[0, \lambda+2]$ to extend the metric $g^{\partial}$ from the boundary $E_{0}=\partial X$ to a positive scalar curvature submersion metric $\bar{g}_{\text {pre }}^{\partial}$ on the total space of this bundle, $\bar{E}_{0}$. To do this, we follow the method described in Lemma 2.7 with respect to the product distribution $\mathcal{H}_{0} \times \mathbb{R}$ to extend $g^{\partial}$ to a psc submersion metric, on $\bar{E}_{0} \cong E_{0} \times[0, \lambda+2]$. We denote the resulting metric, $\bar{g}_{\text {pre }}^{\partial}$. Note, near $E_{0} \times\{\lambda+2\}$, this is a Riemannian cylinder of a pscsubmersion metric over $\left(B_{0}, h_{\text {std }}^{\beta}\right)$ with the fibre $\left(L, \bar{\tau} g_{L}\right)$ for $\bar{\tau}=\bar{\tau}\left(\bar{h}_{\text {pre }}^{\beta}, \mathcal{H}_{0} \times \mathbb{R}\right)$, as defined in $(2.2)$. 

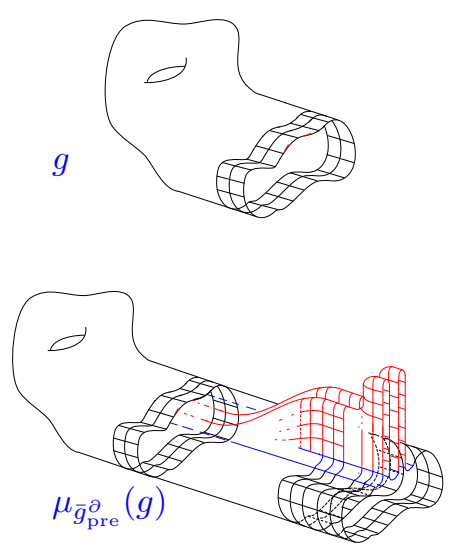

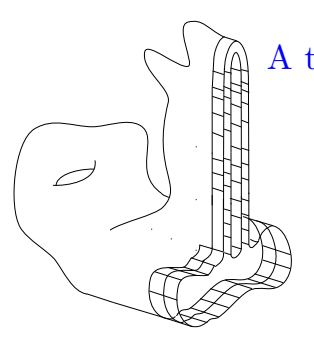

A typical element of $\mathcal{R}_{\mathcal{S}_{1}}^{\mathrm{psc}}\left(X_{1}, \partial X_{1}\right)_{h_{1}^{\beta}}$

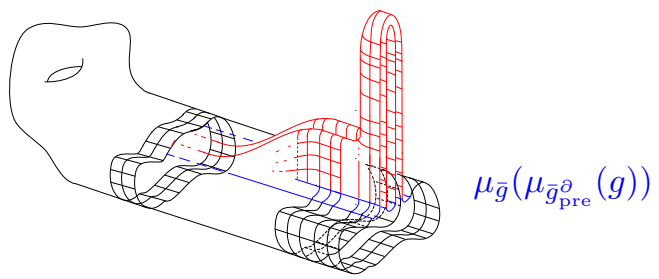

Figure 4. Representative elements of the spaces from the commutative diagram (3.1) above in the case when $L$ is a point.

For any element $g \in \mathcal{R}_{\mathcal{S}}^{\mathrm{psc}}(X, \partial X)_{h_{0}^{\beta}}$, the metric $g \cup_{\partial X} \bar{g}_{\text {pre }}^{\partial}$ on $X \cup \bar{E}_{0}$ (obtained by the obvious gluing) is denoted by $g_{\text {std }}$ and is an element of the space $\mathcal{R}_{\mathcal{S}}^{\mathrm{psc}}(X, \partial X)_{h_{\text {std }}^{\beta}}$. This gives a map

$$
\mu_{\bar{g}_{\mathrm{pre}}^{\partial}}: \mathcal{R}_{\mathcal{S}}^{\mathrm{psc}}(X, \partial X)_{h_{0}^{\beta}} \rightarrow \mathcal{R}_{\mathcal{S}}^{\mathrm{psc}}(X, \partial X)_{h_{\mathrm{std}}^{\beta}}
$$

We denote $\mathcal{R}_{\mathcal{S}-\text { boot }}^{\text {psc }}(X, \partial X)_{h_{\text {std }}^{\beta}}:=\operatorname{Im}\left(\mu_{\bar{g}_{\text {pre }}^{\partial}}\right)$. This new metric is depicted in the bottom left of Figure 4, with the original metric $g$ depicted in the top left. For clarity, this figure depicts only the case when $L$ is a point. Lemma 6.5.5 of [20], consolidating work from previous sections, shows that in the case when $L$ is a point (and so $\partial X=\beta X$ ), the map $\mu_{\bar{g}_{\text {pre }}}$ is a weak homotopy equivalence. This is demonstrated by constructing certain isotopies of psc-metrics on the cylinder $B_{0} \times[0, \lambda+2]$. In our case, where $L$ is not simply a point, we must lift such a compact family of psc-metrics on $B_{0} \times[0, \lambda+2]$ to a corresponding compact family of psc-metrics on $\bar{E}_{0}$. This is done using Lemma 2.7, with respect to the horizontal distribution $\mathcal{H}_{0} \times \mathbb{R}$ and a sufficiently small scaling constant $\bar{\tau}_{\min }>0$.

Turning our attention momentarily to the space $X_{1}=X \cup \bar{E}$, recall that, though suppressed in the notation, the map $\mu_{\left(\bar{B}, \bar{f}, \bar{h}^{\beta}\right)}$ implicitly associates to the bundle, $\bar{p}: \bar{E} \rightarrow \bar{B}$, an admissible horizontal distribution, $\overline{\mathcal{H}}$ extending $\mathcal{H}_{0}$ (earlier we denoted this $\xi\left(\mathcal{H}_{0}\right)$ ).

Consider now an element of the space $\mathcal{R}_{\mathcal{S}-\text { boot }}^{\text {psc }}(X, \partial X)_{h_{\text {std }}^{\beta}}:=\operatorname{Im}\left(\mu_{\bar{g}_{\text {pre }}^{\partial}}\right)$. Such an element has, near the boundary, a "standard piece" where the submersion metric $\bar{g}_{\text {pre }}^{\partial}$ restricts over a region where the base metric takes the form $\mathrm{d} s_{p}^{2}+g_{\text {boot }}^{q+2}(1)_{\Lambda, \bar{l}}$. Replacing this piece, on the base, with $g_{\text {torp }}^{p+1}+g_{\text {torp }}^{q+1}$ near the boundary, determines a base metric on $\bar{B}$. This is exactly what is done in [20, Theorem A], determining a homeomorphism onto its image in the relevant spaces of pscmetrics on the bases. Using Lemma 2.7, with respect to the distribution $\overline{\mathcal{H}}$ allows us to lift the resulting base metric on $\bar{B}$ to a psc-submersion metric on $\bar{E}$. This determines a map

$$
\mu_{\text {Estd }}: \mathcal{R}_{\mathcal{S}-\text { boot }}^{\text {psc }}(X, \partial X)_{h_{\text {std }}^{\beta}} \rightarrow \mathcal{R}_{\mathcal{S}_{1}}^{\text {psc }}\left(X_{1}, \partial X_{1}\right)_{h_{1}^{\beta}}
$$

Replacing the codomain of this map by its image, denoted

$$
\mathcal{R}_{\mathcal{S}_{1}-\text { Estd }}^{\mathrm{psc}}\left(X_{1}, \partial X_{1}\right)_{h_{1}^{\beta}} \subset \mathcal{R}_{\mathcal{S}_{1}}^{\mathrm{psc}}\left(X_{1}, \partial X_{1}\right)_{h_{1}^{\beta}}
$$


we obtain the lower horizontal map in diagram (3.1). A typical element in the image of this map is depicted in the lower right of Figure 4. As with the base case in [20, Theorem A], this lower horizontal map is demonstrably a homeomorphism.

It remains to show that the inclusion $\mathcal{R}_{\mathcal{S}_{1}-\text { Estd }}^{\text {psc }}\left(X_{1}, \partial X_{1}\right)_{h_{1}^{\beta}} \subset \mathcal{R}_{\mathcal{S}_{1}}^{\mathrm{psc}}\left(X_{1}, \partial X_{1}\right)_{h_{1}^{\beta}}$ is a weak homotopy equivalence. Note that the notaiton "Estd" used in describing the former space (originating in [20]) is intended to convey the fact that these metrics take a standard form on a much larger region than typical metrics in $\mathcal{R}_{\mathcal{S}_{1}}^{\mathrm{psc}}\left(X_{1}, \partial X_{1}\right)_{h_{1}^{\beta}}$ and are thus "Extra-standard".

A typical element of $\mathcal{R}_{\mathcal{S}_{1}}^{\mathrm{psc}}\left(X_{1}, \partial X_{1}\right)_{h_{1}^{\beta}}$ (in the case when $L$ is a point and so $\partial X_{1}=\beta X_{1}$ ) is depicted in the upper right of Figure 4. Showing that, in the case when $L$ is a point, a compact family of metrics in $\mathcal{R}_{\mathcal{S}_{1}}^{\mathrm{psc}}\left(X_{1}, \partial X_{1}\right)_{h_{1}^{\beta}}$ could be continuously moved to a compact family of extra standard metrics in $\mathcal{R}_{\mathcal{S}_{1} \text {-Estd }}^{\text {psc }}\left(X_{1}, \partial X_{1}\right)_{h_{1}^{\beta}}$, without moving already extra-standard metrics out of that space, is the most technically difficult part of the proof of [20, Theorem A]. This is done in [20, Section 6.6].

In our case, $L$ is not simply a point. All of the adjustment takes place off of $X$ and so we restrict our attention to $X_{1} \backslash X$. Once again however, the argument in [20, Theorem A] involves continuous deformations of compact families of psc-metrics on the base. Thus, applying Lemma 2.7, for the one admissible horizontal distribution $\overline{\mathcal{H}}$ and a sufficiently small scaling constant $\bar{\tau}_{\min }>0$, all such (compact) deformations of psc-metrics lift to appropriate deformations on the total space $X_{1} \backslash X$. This completes the proof of Theorem 2.17.

\section{Proof of Theorem 1.8}

We now come to the proof of Theorem 1.8. We noted earlier (see Remark 1.9) that part $(i)$ of Theorem 1.8 follows from the main result of Chernysh in [8]. Thus, our focus is on part (ii).

Let $X_{\Sigma}=X \cup_{\partial X}-N(\beta X)$ be a pseudo-manifold as above, where $X$ is a manifold with boundary $\partial X$, the total space of a bundle, $p: \partial X \rightarrow \beta X$ with fibre $L$. This bundle has a structure map $f: \beta X \rightarrow B G$. Recall that $G$ is a subgroup of the isometry group of the simple link $\left(L, g_{L}\right)$. We begin by recalling the restriction map

$$
\text { res: } \mathcal{R}^{\mathrm{psc}}(X, \partial X) \rightarrow \mathcal{R}^{\mathrm{psc}}(\partial X), \quad \text { res: }\left.g \mapsto g\right|_{\partial X} .
$$

As we mentioned earlier, from work of Chernysh [8] and Ebert-Frenck [9], we know that this map is a Serre fibre bundle.

Now we consider two pseudo-manifolds $X_{\Sigma}=X \cup_{\partial X}-N(\beta X)$ and $X_{\Sigma, 1}=X_{1} \cup_{\partial X}-N\left(\beta X_{1}\right)$, where $X_{1}=X \cup_{\partial X} Z$, and the manifold $Z$ is given by an elementary psc-bordism $\bar{B}: \beta X \rightsquigarrow \beta X_{1}$ and a structure map $\bar{f}: \bar{B} \rightarrow B G$, so that $f=\left.\bar{f}\right|_{\beta X}$ and $f_{1}=\left.\bar{f}\right|_{\beta X_{1}}$. Namely, the manifold $Z$ is a total space of the following smooth bundle:

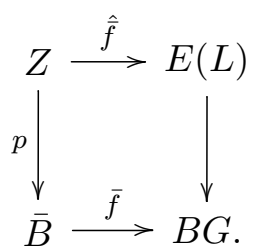

Consider a pair of psc-submersion metrics, $g_{0}^{\partial} \in \mathcal{R}_{\mathcal{S}}^{\mathrm{psc}}(\partial X)$ and $g_{1}^{\partial} \in \mathcal{R}_{\mathcal{S}_{1}}^{\mathrm{psc}}\left(\partial X_{1}\right)$, where $\mathcal{S}_{1}$ is the analogue of the map $\mathcal{S}$ for the bundle $\partial X_{1} \rightarrow \beta X_{1}$. Thus, $g_{0}^{\partial}$ and $g_{1}^{\partial}$ are psc-submersion metrics over $\beta X$ (respectively, over $\beta X_{1}$ ), with fibre metrics $\tau_{0} g_{L}$ and $\tau_{1} g_{L}$ for some constants $\tau_{0}, \tau_{1}>0$. We denote by $h_{0}^{\beta} \in \mathcal{R}^{\mathrm{psc}}(\beta X)$ and $h_{1}^{\beta} \in \mathcal{R}^{\mathrm{psc}}\left(\beta X_{1}\right)$, the respective base metrics $\mathcal{B}\left(g^{\partial}\right)=h^{\beta}=: h_{0}^{\beta}$ and $\mathcal{B}_{1}\left(g_{1}^{\partial}\right)=: h_{1}^{\beta}$. 
Now we notice that the spaces $\mathcal{R}_{\mathcal{S}}^{\mathrm{psc}}(X, \partial X)_{h_{0}^{\beta}}$ and $\mathcal{R}_{\mathcal{S}}^{\mathrm{psc}}\left(X_{1}, \partial X_{1}\right)_{h_{1}^{\beta}}$ coincide with the fibres $\mathcal{R}_{\mathcal{S}}^{\mathrm{psc}}(X, \partial X)_{h_{0}^{\beta}}=\operatorname{res}_{0}^{-1}\left(g_{0}^{\partial}\right), \quad \mathcal{R}_{\mathcal{S}}^{\mathrm{psc}}\left(X_{1}, \partial X_{1}\right)_{h_{1}^{\beta}}=\operatorname{res}_{1}^{-1}\left(g_{1}^{\partial}\right)$,

of the corresponding restriction maps:

$\operatorname{res}_{0}: \mathcal{R}^{\mathrm{psc}}(X, \partial X) \rightarrow \mathcal{R}^{\mathrm{psc}}(\partial X), \quad \operatorname{res}_{1}: \mathcal{R}^{\mathrm{psc}}\left(X_{1}, \partial X_{1}\right) \rightarrow \mathcal{R}^{\mathrm{psc}}\left(\partial X_{1}\right)$.

We consider the inclusion map

$$
\mathcal{R}_{\mathcal{S}}^{\mathrm{psc}}(\partial X) \hookrightarrow \mathcal{R}^{\mathrm{psc}}(\partial X) .
$$

Now, by definition, we obtain the space $\mathcal{R}^{\text {psc }}\left(X_{\Sigma}\right)$ as a pull-back in the following diagram

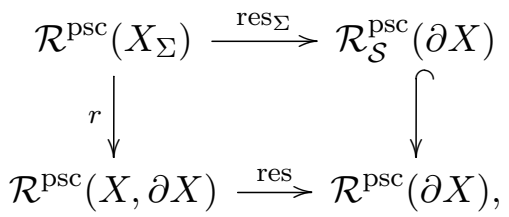

where the right vertical map is inclusion. The left vertical map sends a psc-metric on $X_{\Sigma}$ to its restriction to $X$.

There are two more maps we must define. Recall we have a projection map,

$$
\mathcal{B}: \mathcal{R}_{\mathcal{S}}^{\mathrm{psc}} \rightarrow \mathcal{R}^{\mathrm{psc}}(\beta X),
$$

sending each submersion metric to its base. In Lemma 2.5 we showed that $\mathcal{B}$ forms part of a homotopy equivalence. In particular the lifting map (2.5)

$$
\mathcal{S}_{\mathcal{H}}^{\mathrm{psc}}: \mathcal{R}^{\mathrm{psc}}(\beta X) \rightarrow \mathcal{R}_{\mathcal{S}}^{\mathrm{psc}},
$$

for any choice of horizontal distribution, $\mathcal{H}$, on the bundle $p: \partial X \rightarrow \beta X$, is a homotopy inverse. Composing, for some choice of $\mathcal{H}$, the map $\mathcal{S}_{\mathcal{H}}^{\text {psc }}$ with the inclusion map above yields the map

$$
\pi_{\mathcal{H}}: \mathcal{R}^{\mathrm{psc}}(\beta X) \stackrel{\mathcal{S}_{\mathcal{H}}^{\mathrm{psc}}}{\longrightarrow} \mathcal{R}_{\mathcal{S}}^{\mathrm{psc}}(\partial X) \hookrightarrow \mathcal{R}^{\mathrm{psc}}(\partial X) .
$$

The restriction map $r: \mathcal{R}^{\text {psc }}\left(X_{\Sigma}\right) \rightarrow \mathcal{R}^{\text {psc }}(X, \partial X)$ above is in fact a homeomorphism onto its image, $\mathcal{R}_{\mathcal{S}}^{\mathrm{psc}}(X, \partial X)$, the space of well adapted psc-metrics on $X$. Replacing the codomain $\mathcal{R}^{\mathrm{psc}}(X, \partial X)$ with $\mathcal{R}_{\mathcal{S}}^{\mathrm{psc}}(X, \partial X)$, we then compose $r$ with the restrictions res and $\mathcal{B}$ to obtain the map

$$
\operatorname{res}_{\Sigma}: \mathcal{R}^{\mathrm{psc}}\left(X_{\Sigma}\right) \stackrel{r}{\rightarrow} \mathcal{R}_{\mathcal{S}}^{\mathrm{psc}}(X, \partial X) \stackrel{\text { res }}{\longrightarrow} \mathcal{R}_{\mathcal{S}}^{\mathrm{psc}}(\partial X) \stackrel{\mathcal{B}}{\rightarrow} \mathcal{R}^{\mathrm{psc}}(\beta X) .
$$

As $r$ (as deployed above) is now a homeomorphism, res is a Serre fibre bundle and $\mathcal{B}$ is a homotopy equivalence, we conclude that the composition map, ress, is a Serre fibre bundle. Recalling that the spaces $\mathcal{R}^{\mathrm{psc}}(X, \partial X)_{g_{0}^{\partial}}=\operatorname{res}_{0}^{-1}\left(g_{0}^{\partial}\right)$ and $\mathcal{R}_{\mathcal{S}}^{\mathrm{psc}}(X, \partial X)_{h_{0}^{\beta}}$ are in fact the same, we obtain the following diagram of fibre bundles:

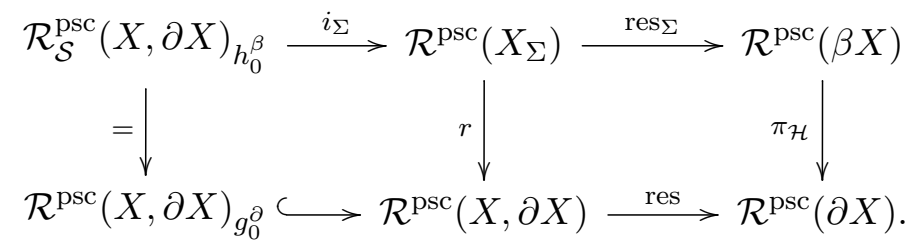

Here, the top left horizontal map $i_{\Sigma}$ is the metric attaching map defined in 2.6. 
Let $\left(\bar{B}, \bar{h}_{\beta}\right):\left(\beta X, h_{0}^{\beta}\right) \rightsquigarrow\left(\beta X_{1}, h_{1}^{\beta}\right)$ be an elementary psc-bordism (with $\left.p, q \geq 2\right)$, which is given together with a map $\bar{f}: \bar{B} \rightarrow B G$ such that $f=\left.\bar{f}\right|_{\beta X}$ and $f_{1}=\left.\bar{f}\right|_{\beta X_{1}}$. In turn, the psc-bordism $\left(\bar{B}, \bar{h}_{\beta}\right)$ determines a corresponding psc-bordism $\left(Z, \bar{g}^{\partial}\right):\left(\partial X, g_{0}^{\partial}\right) \rightsquigarrow\left(\partial X_{1}, g_{1}^{\partial}\right)$ by means of Lemma 2.7. In particular, the psc-submersion metrics $g_{0}^{\partial}$ and $g_{1}^{\partial}$ have base metrics $h_{0}^{\beta}$ and $h_{1}^{\beta}$. Theorem 2.2 and Theorem 2.17 give us the following homotopy equivalences:

$$
\begin{aligned}
& \mu_{\bar{B}, \bar{g}^{\beta}}: \mathcal{R}^{\mathrm{psc}}(\beta X) \stackrel{\simeq}{\longrightarrow} \mathcal{R}^{\mathrm{psc}}\left(\beta X_{1}\right), \\
& \mu_{Z, \bar{g}^{\beta}}: \mathcal{R}^{\mathrm{psc}}(\partial X) \stackrel{\simeq}{\longrightarrow} \mathcal{R}^{\mathrm{psc}}\left(\partial X_{1}\right), \\
& \mu_{Z, \bar{g}^{\partial}}: \mathcal{R}^{\mathrm{psc}}(X, \partial X)_{g^{\partial}} \stackrel{\simeq}{\longrightarrow} \mathcal{R}^{\mathrm{psc}}\left(X_{1}, \partial X_{1}\right)_{g_{1}^{\partial}}, \\
& \mu_{\bar{B}, \bar{f}, \bar{h}^{\beta}}: \quad \mathcal{R}_{\mathcal{S}}^{\mathrm{psc}}(X, \partial X)_{h^{\beta}} \stackrel{\simeq}{\longrightarrow} \mathcal{R}_{\mathcal{S}_{1}}^{\mathrm{psc}}\left(X_{1}, \partial X_{1}\right)_{h_{1}^{\beta}} .
\end{aligned}
$$

We obtain the following commutative diagram:

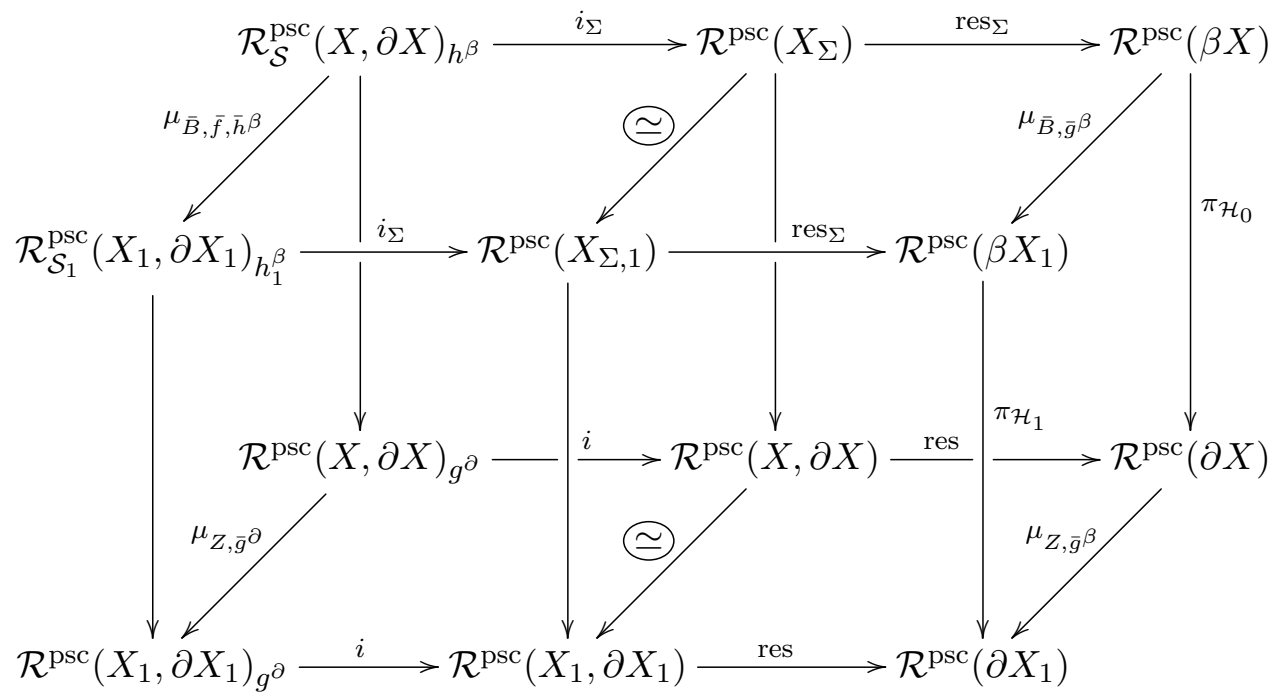

where all horizontal rows are Serre fibre bundles, and the maps $\pi_{\mathcal{H}_{0}}$ and $\pi_{\mathcal{H}_{1}}$ are defined as above. Recall in particular that the left most vertical arrows (front and back) represent equality. Commutativity of this diagram makes it evident that the circled maps above are weak homotopy equivalences. This proves Theorem 1.8.

\section{Some further developments}

In this section we would like to emphasize that recent results concerning homotopy groups of the spaces, $\mathcal{R}^{\mathrm{psc}}(M)$, of psc-metrics on a smooth closed manifold $M$ (see [4, 10, 11, 13, 14]) could be applied directly and indirectly to the case of manifolds with $(L, G)$-fibered singularities. In particular, we would like to attract the attention of topologically-minded experts to relevant conjectures and results from the recent work $[5,6]$.

\subsection{Index-difference map}

We mentioned earlier that the homotopy-invariance of various spaces of psc-metrics is a crucial property in helping detect their non-trivial homotopy groups. With this in mind, there is a secondary index invariant, the index-difference map

$$
\text { inddiff }_{g_{0}}: \mathcal{R}^{\mathrm{psc}}(M) \rightarrow \Omega^{\infty+n+1} \mathbf{K O},
$$


which is defined as follows. Let $g_{0} \in \mathcal{R}^{\text {psc }}(M)$ be a base point. Then for any psc-metric $g$ on $M$, there is an interval $g_{t}=(1-t) g_{0}+t g$ of metrics such that a corresponding curve of the Dirac operators $D_{g_{t}}$ starts and ends at the subspace $\left(\text { Fred }^{n}\right)^{\times} \subset$ Fred $^{n}$ of invertible Dirac operators. Since the subspace $\left(\text { Fred }^{n}\right)^{\times}$is contractible, the curve $D_{g_{t}}$ is a loop in the space Fred $^{n}$ of all Dirac operators. This space, in turn, is homotopy equivalent to the loop space $\Omega^{\infty+n} \mathbf{K O}$ representing a shifted $K O$-theory, i.e., $\pi_{q}\left(\Omega^{\infty+n} \mathbf{K O}\right)=K O_{n+q}$. Thus the curve $D_{g_{t}}$ gives an element in $\Omega^{\infty+n+1} \mathbf{K O}$, well-defined up to homotopy, to determine the map (5.1).

Theorem 5.1 (Botvinnik-Ebert-Randal-Williams [4], and Perlmutter [13, 14]). Assume $M$ is a spin manifold with $\operatorname{dim} M \geq 5$ and $\mathcal{R}^{\mathrm{psc}}(M) \neq \varnothing$ with a base point $g_{0} \in \mathcal{R}^{\mathrm{psc}}(M)$. Then the index-diffence map (5.1) induces a non-trivial homomorphism in the homotopy groups

$$
\left(\text { inddiff }_{g_{0}}\right)_{*}: \pi_{q}\left(\mathcal{R}^{\mathrm{psc}}(M)\right) \rightarrow K O_{q+n+1}
$$

when the target group $K O_{q+n+1}$ is non-trivial.

\subsection{Results and conjectures}

The reader should note that much is also known about the spaces of psc-metrics for non-simply connected manifolds; see $[10,11]$. We will however return to the same examples we considered above. We have the following conjectures concerning examples (1) and (2):

Conjecture 5.2. Let $X_{\Sigma}$ be a spin $(\langle k\rangle$-fb)-manifold. Assume $\operatorname{dim} X \geq 7$ and $X$ and $\beta X \neq \varnothing$ are simply-connected and $\mathcal{R}^{\mathrm{psc}}\left(X_{\Sigma}\right) \neq \varnothing$ with a base point $g_{0} \in \mathcal{R}^{\mathrm{psc}}\left(X_{\Sigma}\right)$. Then there is an index-difference map

$$
\text { inddiff }_{g_{0}}^{\langle k\rangle}: \mathcal{R}^{\mathrm{psc}}\left(X_{\Sigma}\right) \rightarrow \Omega^{\infty+n+1} \mathbf{K} \mathbf{O}^{\langle k\rangle},
$$

which induces a non-trivial homomorphism in the homotopy groups

$$
\left(\operatorname{inddiff}_{g_{0}}^{\langle k\rangle}\right)_{*}: \pi_{q}\left(\mathcal{R}^{\mathrm{psc}}\left(X_{\Sigma}\right)\right) \rightarrow K O_{n+q+1}^{\langle k\rangle}
$$

when the target group $K O_{n+q+1}^{\langle k\rangle}\left(K O\right.$ with $\mathbb{Z}_{k}$-coefficients) is non-trivial.

Conjecture 5.3. Let $X_{\Sigma}$ be a spin manifold with ( $\eta$-fb)-singularity of dimension $n \geq 9$. Assume $\beta X \neq \varnothing$, and $\mathcal{R}^{\mathrm{psc}}\left(X_{\Sigma}\right) \neq \varnothing$ with a base point $g_{0} \in \mathcal{R}^{\mathrm{psc}}\left(X_{\Sigma}\right)$. Then there is an index-difference map

$$
\text { inddiff } g_{0}^{\eta-\mathrm{fb}}: \mathcal{R}^{\mathrm{psc}}\left(X_{\Sigma}\right) \rightarrow \Omega^{\infty+n+1} \mathbf{K} \mathbf{O}^{\eta-\mathrm{fb}},
$$

which induces a non-trivial homomorphism in the homotopy groups

$$
\left(\text { inddiff }_{g_{0}}^{\eta}\right)_{*}: \pi_{q}\left(\mathcal{R}^{\mathrm{psc}}\left(X_{\Sigma}\right)\right) \rightarrow K O_{q+n+1}^{\eta-\mathrm{fb}}
$$

when the target group $K O_{q+n+1}^{\eta-\mathrm{fb}}=K O_{q+n+1}\left(\mathbb{C P}^{\infty}\right)$ is non-trivial.

It turns out that the above examples (3) and (4) (and many others; see [5]) lead to particular results concerning the homotopy groups of the spaces $\mathcal{R}^{\mathrm{psc}}\left(X_{\Sigma}\right)$. Let $X_{\Sigma}=X \cup_{\partial X}-N(\beta X)$ be a spin manifold with $(L, G)$-singularities. Let $g \in \mathcal{R}^{\mathrm{psc}}\left(X_{\Sigma}\right)$ be a well-adapted metric. Then $g$ determines the metrics $g_{\partial X} \in \mathcal{R}^{\mathrm{psc}}(\partial X)$ and $g_{\beta X} \in \mathcal{R}^{\mathrm{psc}}(\beta X)$ such that the bundle $\partial X \rightarrow \beta X$ is a Riemannian submersion. We fix the metric $g_{\beta X, 0}$. This gives rise to a Serre fibre bundle

$$
\operatorname{res}_{\Sigma}: \mathcal{R}^{\mathrm{psc}}\left(X_{\Sigma}\right) \rightarrow \mathcal{R}^{\mathrm{psc}}(\beta X)
$$

with fibre $\mathcal{R}^{\mathrm{psc}}\left(X_{\Sigma}\right)_{g_{\beta X}, 0}$, where $\mathcal{R}^{\mathrm{psc}}\left(X_{\Sigma}\right)_{g_{\beta X}}$ is the space of all metrics $g \in \mathcal{R}^{\mathrm{psc}}\left(X_{\Sigma}\right)$ which restrict to $g_{\beta X, 0}$ on $\mathcal{R}^{\mathrm{psc}}(\beta X)$. Since the metric $g_{\partial X, 0}$ on $\partial X$ is determined by the metric $g_{\beta X, 0}$, the fibre $\mathcal{R}^{\mathrm{psc}}\left(X_{\Sigma}\right)_{g_{\beta X}, 0}$ coincides with the space $\mathcal{R}^{\mathrm{psc}}(X)_{g_{\partial X}, 0}$. Here is the result we need: 
Theorem 5.4 (see [6, Theorem 6.1]). Let $X_{\Sigma}$ be an (L,G)-fibred compact pseudo-manifold with $L$ a simply connected homogeneous space of a compact semisimple Lie group. Assume $\mathcal{R}^{\mathrm{psc}}\left(X_{\Sigma}\right) \neq \varnothing$. Then there exists a section $s: \mathcal{R}^{\mathrm{psc}}(\beta X) \rightarrow \mathcal{R}^{\mathrm{psc}}\left(X_{\Sigma}\right)$ to res$\Sigma$. In particular, there is a split short exact sequence:

$$
0 \rightarrow \pi_{q}\left(\mathcal{R}_{w}^{+}\left(X_{\Sigma}\right)_{g_{\beta X}}\right) \stackrel{i_{*}}{\rightarrow} \pi_{q}\left(\mathcal{R}_{w}^{+}\left(X_{\Sigma}\right)\right) \stackrel{\left(\mathrm{res}_{\Sigma}\right)_{*}}{\longrightarrow} \pi_{q}\left(\mathcal{R}^{+}(\beta X)\right) \rightarrow 0, \quad q=0,1, \ldots
$$

Here is one of the conclusions we would like to emphasize:

Corollary 5.5 (see [5, Corollary 6.7]). Let $X_{\Sigma}$ be an $(L, G)$-fibred compact pseudo-manifold with $L$ a simply connected homogeneous space of a compact semisimple Lie group, and $n-\ell-1 \geq 5$, where $\operatorname{dim} X=n$, $\operatorname{dim} L=\ell$. Let $g_{0} \in \mathcal{R}^{\mathrm{psc}}\left(X_{\Sigma}\right) \neq \varnothing$ be a base point giving corresponding base points, the metrics $g_{\beta X, 0} \in \mathcal{R}^{\mathrm{psc}}(\beta X), g_{\partial X, 0} \in \mathcal{R}^{\mathrm{psc}}(\partial X)$ and $g_{X, 0} \in \mathcal{R}^{\mathrm{psc}}(X)_{g_{\partial X, 0}}$.

If $M_{\Sigma}$ is spin and simply connected, then we have the following commutative diagram:

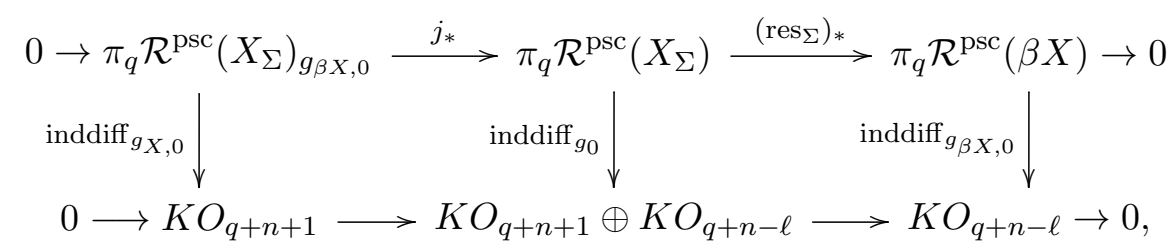

where the homomorphisms inddiff $g_{X, 0}$ and inddiff $g_{\beta X, 0}$ are both nontrivial whenever the target groups are. In particular, the homomorphism

$$
\text { inddiff } g_{0}: \pi_{q} \mathcal{R}^{\mathrm{psc}}\left(X_{\Sigma}\right) \rightarrow K O_{q+n+1} \oplus K O_{q+n-\ell}
$$

is surjective rationally and surjective onto the torsion of $K O_{q+n+1} \oplus K O_{q+n-\ell}$.

There are much more general results concerning the homotopy groups of the space $\pi_{q} \mathcal{R}^{\mathrm{psc}}\left(X_{\Sigma}\right)$ if $X_{\Sigma}$ is not simply-connected; see [5, Section 6].

\section{Appendix: cone metrics}

\subsection{Cone metric of $\left(L, g_{L}\right)$ when $g_{L}$ has constant non-negative scalar curvature}

Let $L$ be smooth closed manifold of dimension $l$ and $g_{L}$, a metric on $L$ with constant non-negative scalar curvature. We wish to extend $g_{L}$ to a scalar flat metric on the cone $C(L)$. The resulting metric will be denoted $g_{C(L)}$ and is constructed as follows. Consider the warped product metric $\mathrm{d} t^{2}+\phi(t)^{2} g_{L}$ on the cylinder $(0, b) \times L$ for some smooth function $\phi:[0, b] \rightarrow(0, \infty)$. The scalar curvature, $s$, of such a metric is given by the formula

$$
s=\frac{-4 l}{(l+1)}\left(\frac{u^{\prime \prime}}{u}\right)+s_{g_{L}} u^{\frac{-4}{l+1}},
$$

where $l:=\operatorname{dim} L, u$ is the function satisfying $u^{\frac{2}{l+1}}=\phi$ and $s_{g_{L}}$ is the scalar curvature of the metric $g_{L}$.

Let us first consider the case when $s_{g_{L}}=0$. An easy calculation shows $s=0$ precisely when

$$
u(t)=A t+B
$$

for various constants $A, B$. Indeed, in the case when $A=0$, we obtain a Riemannian cylinder. In our case, we set $A=1, B=0$ and $b=\frac{1}{2}$. This gives rise to a metric

$$
g_{C(L)}=\mathrm{d} t^{2}+t^{\frac{4}{l+1}} g_{L},
$$


on the cylinder $\left[0, \frac{1}{2}\right] \times L$, which collapses at $t=0$. The result is a scalar-flat metric (with a singularity) on the cone $C(L)$ obtained from $\left[0, \frac{1}{2}\right] \times L$ by collapsing $\{0\} \times L$ to a point.

Now consider the case that the constant $s_{g_{L}}>0$. In this case, we set $\phi(t)=\frac{1}{c_{L}} t$, where the constant $c_{L}$ satisfies

$$
c_{L}=\sqrt{\frac{l(l-1)}{s_{g_{L}}}} .
$$

Thus $u=t^{\frac{l+1}{2}}$ and the resulting metric

$$
g_{C(L)}=\mathrm{d} t^{2}+\frac{1}{c_{L}^{2}} t^{2} g_{L}
$$

has scalar curvature given by

$$
s_{g_{C(L)}}=\left(c_{L}^{2} s_{g_{L}}-l(l-1)\right) t^{-2}=0,
$$

given our choice of $c_{L}$. Again, we may assume that $t \in\left[0, \frac{1}{2}\right]$.

Remark 6.1. Regarding the earlier setting when $g_{L}$ is scalar flat, we will typically only be interested in the case when $L=S^{1}$ and so $l=1$. Thus, metric formulae in both cases coincide.

\subsection{Attaching the cone to the cylinder}

In constructing certain metrics on $X_{\Sigma}$, it is necessary to attach the above conical metric, $g_{C(L)}$, to a cylindrical metric $\mathrm{d} t^{2}+g_{L}$. This requires an intermediary "attaching" metric to transition smoothly between the two forms. To do this, we first specify a smooth transition function, $a:[0,1] \rightarrow\left[0, \frac{1}{2}\right]$, satisfying:

(i) $a(t)=\frac{1}{2}+t$ when $t$ is near 0 ,

(ii) $a(t)=1$ when $t$ is near 1 ,

(iii) for all $t \in[0,1], 0 \leq a^{\prime}(t) \leq 1$ and $a^{\prime \prime}(t) \leq 0$.

The existence of such functions is obvious. We choose one and define the attaching metric, $g_{\text {att }(L)}$, on the cylinder $[0,1] \times L$ by the formula:

$$
g_{\operatorname{att}(L)}= \begin{cases}\mathrm{d} t^{2}+a^{\frac{4}{l+1}}(t) g_{L}, & s_{g_{L}}=0 \\ \mathrm{~d} t^{2}+a^{2}(t) g_{L}, & s_{g_{L}}>0\end{cases}
$$

Proposition 6.2. Attaching $\left(\left[0, \frac{1}{2}\right] \times L, g_{C(L)}\right)$ to $\left([0,1] \times L, g_{\operatorname{att}(L)}\right)$ by identifying $\left\{\frac{1}{2}\right\} \times L$ in the former with $\{0\} \times L$ in the latter determines a smooth metric of non-negative scalar curvature $g_{\text {att }(L)} \cup g_{C(L)}$.

Proof. Smoothness is immediate from the properties of the transition function $a$. The component, $g_{C(L)}$, has zero scalar curvature and that $g_{\text {att }(L)}$ has non-negative scalar curvature is easily seen from the formula (6.1).

\section{Acknowledgments}

BB was partially supported by Simons collaboration grant 708183 . We thank the referees for their careful reading and thoughtful comments. The second author would like to thank David Wraith for some productive conversations. 


\section{References}

[1] Bérard-Bergery L., Scalar curvature and isometry group, in Spectra of Riemannian Manifolds, Kaigai Publications, Tokyo, 1983, 9-28.

[2] Besse A.L., Einstein manifolds, Ergebnisse der Mathematik und ihrer Grenzgebiete (3), Vol. 10, SpringerVerlag, Berlin, 1987.

[3] Botvinnik B., Manifolds with singularities accepting a metric of positive scalar curvature, Geom. Topol. 5 (2001), 683-718, arXiv:math.DG/9910177.

[4] Botvinnik B., Ebert J., Randal-Williams O., Infinite loop spaces and positive scalar curvature, Invent. Math. 209 (2017), 749-835, arXiv:1411.7408.

[5] Botvinnik B., Piazza P., Rosenberg J., Positive scalar curvature on simply connected spin pseudomanifolds, arXiv:1908.04420.

[6] Botvinnik B., Piazza P., Rosenberg J., Positive scalar curvature on spin pseudomanifolds: the fundamental group and secondary invariants, arXiv:2005.02744.

[7] Botvinnik B., Rosenberg J., Positive scalar curvature on manifolds with fibered singularities, arXiv:1808.06007.

[8] Chernysh V., On the homotopy type of the space $\mathcal{R}^{+}(M)$, arXiv:math.GT/0405235.

[9] Ebert J., Frenck G., The Gromov-Lawson-Chernysh surgery theorem, arXiv:1807.06311.

[10] Ebert J., Randal-Williams O., Infinite loop spaces and positive scalar curvature in the presence of a fundamental group, Geom. Topol. 23 (2019), 1549-1610, arXiv:1711.11363.

[11] Ebert J., Randal-Williams O., The positive scalar curvature cobordism category, arXiv:1904.12951.

[12] Gromov M., Lawson Jr. H.B., The classification of simply connected manifolds of positive scalar curvature, Ann. of Math. 111 (1980), 423-434.

[13] Perlmutter N., Cobordism categories and parametrized Morse theory, arXiv:1703.01047.

[14] Perlmutter N., Parametrized Morse theory and positive scalar curvature, arXiv:1705.02754.

[15] Stolz S., Simply connected manifolds of positive scalar curvature, Ann. of Math. 136 (1992), 511-540.

[16] Tuschmann W., Wraith D.J., Moduli spaces of Riemannian metrics, Oberwolfach Seminars, Vol. 46, Birkhäuser Verlag, Basel, 2015.

[17] Walsh M., Metrics of positive scalar curvature and generalised Morse functions, Part I, Mem. Amer. Math. Soc. 209 (2011), xviii+80 pages, arXiv:0811.1245.

[18] Walsh M., Cobordism invariance of the homotopy type of the space of positive scalar curvature metrics, Proc. Amer. Math. Soc. 141 (2013), 2475-2484, arXiv:1109.6878.

[19] Walsh M., $H$-spaces, loop spaces and the space of positive scalar curvature metrics on the sphere, Geom. Topol. 18 (2014), 2189-2243, arXiv:1301.5670.

[20] Walsh M., The space of positive scalar curvature metrics on a manifold with boundary, New York J. Math. 26 (2020), 853-930, arXiv:1411.2423. 